\title{
Existência de Atratores Globais para Equações Hiperbólicas O caso do Crescimento Subcrítico em Domínios Tridimensionais-
}

\author{
Cláudia Buttarello Gentile
}

Orientador: Prof. Dr. Alexandre Nolasco de Carvalho

Dissertação apresentada ao Instituto de Ciências Matemáticas de São Carlos - USP, como parte dos requisitos para obtenção do título de "Mestre em Ciências - Área: Matemática".

USP - São Carlos

Dezembro de 1994 
"O entendimento dos símbolos e dos rituais (simbólicos) exige do intérprete que possua cinco qualidades ou condiçōes, sem as quais os símbolos serão para ele mortos e ele um morto para eles.

A primeira é a simpatia; não direi a primeira em tempo, mas conforme vou citando, e cito por graus de simplicidade. Tem o intérprete que sentir simpatia pelo símbolo que se propōe interpretar. A atitude cauta, a irônica, a deslocada - todas elas privam o intérprete da primeira condição para poder interpretar.

A segunda é a intuição. A simpatia pode auxiliá-la, se ela já existe, porén não criá-la. Por intuição se entende aquela espécie de entendimento com que se sente o que está além do símbolo, sem que se veja.

A terceira é a inteligência. A inteligência analisa, decompöe, reconstrói noutro nivel o símbolo; tem, porém que fazê-lo depois que, no fundo, é tudo o mesmo. Nāo direi erudiçāo, como poderia no exame dos símbolos, é o de relacionar no alto o que está de acordo com a relação que está embaixo. Nào poderá fazer isso se a simpatia não tiver lembrado essa relação, se a intuiçāo a nāo tiver estabelecido. Então a inteligência, de discursiva que naturalmente é, se tornará analógica, e o símbolo poderá ser interpretado.

A quarta é a compreensão, entendendo por esta palavra o conhecimento de outras matérias, que permitam o símbolo seja iluminado por várias luzes, relacionado com vários outros símbolos, pois que, no fundo, é tudo o mesmo. Náo direi erudiçáo, como poderia ter dito, pois a erudição é uma soma; nem direi cultura, pois a cultura é uma síntese; e a compreensão é uma vida. Assim certos símbolos não podem ser bem entendidos se não houver antes, ou no mesmo tempo, o entendimento de simbolos diferentes.

A quinta é a menos definivel. Direi talvez, falando a uns, que é a graça, falando a outros, que é a mão do Superior Incógnito, falando a terceiros, que é o Conhecimento e Conversação do Santo Anjo da Guarda, entendendo cada uma destas coisas, que são a mesma da maneira como as entendem aqueles que delas usam, falando ou escrevendo."

Fernando Pessoa 
Aos meus pais

Geraldo e Janair 


\section{Agradecimentos ${ }^{1}$}

Agradeço ao Alexandre pela firmeza, pela disponibilidade e sobretudo pela paciência, ao Marcão principalmente pela força que me deu na ocasião do meu exame de qualificação, aos professores do ICMSC dos quais tive o prazer de ser aluna, aos professores da graduação que me apoiaram e incentivaram apesar das minhas hesitações, e em especial ao professor Henrique Lazari, pois foi sob a sua orientação que eu aprendi a aprender matemática.

Agradeço ainda aos meus pais por terem, muitas vezes em detrimento de si, possibilitado os meus estudos, aos meus irmãos Carla, Érika, Marco, Chico e Carol pelo apoio incondicional e pela forte amizade que nos une, a todos os bons amigos de dentro e de fora do departamento, particularmente à Sandra, pelo companheirismo nestes dois anos de São Carlos, e ao Valente, pela cumplicidade que às vezes me permite esquecer que a solidão é inerente ao homem.

\footnotetext{
${ }^{1}$ Este trabalho teve suporte financeiro do $\mathrm{CNPq}$
} 


\begin{abstract}
In this work we prove the existence of global attractors for the scalar linearly damped hyperbolic equation in a bounded smooth domain $\Omega \subset \mathbb{R}^{3}$

$$
\begin{gathered}
w_{t t}+\beta w_{t}=\Delta w+f(w) \text { in } \Omega, \\
\omega=0 \quad \text { in } \partial \Omega,
\end{gathered}
$$
\end{abstract}

when the nonlinear term grows subcritically . 


\section{Resumo}

Neste trabalho nós provamos a existência de atratores globais para equações escalares hiperbólicas com atrito linear em domínios suaves $\Omega \subset \mathbb{R}^{3}$

$$
\begin{gathered}
w_{t t}+\beta w_{t}=\Delta w+f(w) \quad \text { em } \Omega, \\
\omega=0 \quad \text { em } \partial \Omega,
\end{gathered}
$$

com o termo não linear satisfazendo uma condição de crescimento subcrítico. 


\section{Conteúdo}

1 Preliminares 3

1.1 Semigrupos e Potências Fracionárias . . . . . . . . . . . . 3

1.2 Existência, Unicidade e Regularidade . . . . . . . . . . . . 9

1.3 Espaços de Sobolev . . . . . . . . . . . . . . . . . . . 14

2 Atratores para semigrupos e equações de evolução 28

3 Atratores Globais $\quad 39$

3.1 Introdução . . . . . . . . . . . . . . . . . . . 39

3.2 Existência Local de Soluções . . . . . . . . . . . . . . . . . . 39

3.3 Existência Global e Limitação . . . . . . . . . . . . . . . . . . 45

3.4 Compacidade Assintótica e Existência de Atratores Globais . . . 49 


\section{Introdução}

Seja $\Omega$ um subconjunto aberto, limitado de $\mathbb{R}^{3}$, com fronteira $\partial \Omega$ suave e considere o seguinte problema de valor inicial e fronteira

$$
\begin{aligned}
& u_{t t}+\beta u_{t}=\Delta u+f(u) \text { em } \Omega, \\
& u=0, \text { em } \partial \Omega .
\end{aligned}
$$

Neste trabalho procuramos obter condições ótimas de crescimento para o termo não linear $f$ sob as quais o problema (0.1) tenha um atrator global minimal, e de forma a não utilizar na prova da existência do mesmo a fórmula da variação das constantes não linear de Alekseev que requer que o semigrupo associado a (0.1) seja um $C^{\mathbf{1}}$-semigrupo (veja Arrieta, Carvalho e Hale [1992]).

Como é usual assumimos que a função $f: \mathbb{R} \rightarrow \mathbb{R}$ satisfaça a seguinte condição de dissipação

$$
\limsup _{|u| \rightarrow \infty} \frac{f(u)}{u} \leq-\delta<0
$$

mas poderíamos assumir

$$
\limsup _{|u| \rightarrow \infty} \frac{f(u)}{u} \leq 0
$$

sem prejuízo de qualquer resultado.

A condição de crescimento adotada é a condição chamada de subcrítica que é

$$
\lim _{|u| \rightarrow \infty} \frac{f^{\prime}(u)}{u^{2}}=0
$$

A técnica utilizada na prova da existência de um atrator global para o problema (0.1) com termo não linear $f$ satisfazendo $(0.2)$ e $(0.4)$ coincide com a técnica utilizada para o caso não crítico

$$
\left|f^{\prime}(u)\right| \leq c\left(1+|u|^{\gamma}\right), \gamma<2
$$


em todos os pontos, com exceção da prova de que o termo não linear é compacto, onde utilizamos uma técnica parecida com a adotada em Arrieta, Carvalho e Hale [1992], decompondo a não linearidade em uma parte compacta e uma não compacta. No caso de crescimento subcrítico, contudo, esta decomposição pode ser feita com a parte não compacta tão pequena quanto se queira, possibilitando provar que esta não linearidade é ainda compacta.

Este trabalho está organizado da seguinte forma. No Capítulo 1 introduzimos os conceitos básicos indispensáveis ao desenvolvimento do trabalho, muitos dos quais sem prova. No Capítulo 2 apresentamos uma completa descrição de como obter existência de atratores globais para semigrupos, com todas as provas incluídas. Finalmente, no Capítulo 3 utilizamos os resultados dos capítulos precedentes para mostrar a existência de atratores globais para o problema $(0.1)$ com as condições de crescimento e dissipação acima. 


\section{Capítulo 1}

\section{Preliminares}

\subsection{Semigrupos e Potências Fracionárias}

Seja $X$ um espaço de Banach real ou complexo e $L(X)$ o espaço das transformações lineares e contínuas de $X$ em $X$ com a norma usual

$$
\|T\|=\sup _{\substack{x \neq 0 \\ x \in X}} \frac{\|T x\|_{X}}{\|x\|_{X}} .
$$

Definição 1.1 Um semigrupo de operadores lineares limitados em X é uma família $\{T(t), 0 \leq t<\infty\} \subset L(X)$, satisfazendo:

- $T(0)=I$;

- $T(t+s)=T(t) T(s) \quad \forall t, s \geq 0$.

$T(t)$ é fortemente contínuo ou um $C_{0}$ semigrupo se

$$
\lim _{t \downarrow 0} T(t) x=x, \quad \forall x \in X
$$

Definição $1.2 O$ gerador infinitesimal de um $C_{0}$ semigrupo $T(t)$ é o operador $A: \mathbf{D}(A) \subset X \rightarrow X$, definido por

$$
A x=\lim _{t \downarrow 0} \frac{T(t) x-x}{t}, \quad \forall x \in \mathbf{D}(A),
$$

e o domínio de $A, \mathbf{D}(A)$, é o conjunto de todos os elementos $x \in X$ para os quais este limite existe. 
O gerador infinitesimal de um $C_{0}$ semigrupo é único, e se $T(t)$ e $S(t)$ são $C_{0}$ semigrupos com gerador infinitesimal $A$ e $B$ respectivamente, $A=B$ implica $T(t)=S(t)$, $\forall t \geq 0$.

Consideremos o problema de Cauchy

$$
\left\{\begin{aligned}
\frac{d T(t)}{d t} & =A T(t) \\
T(0) & =I
\end{aligned}\right.
$$

onde $A$ é um operador linear limitado. A solução de (1.1) é $T(t)=e^{A t}$ que satisfaz as condições da Definição 1.1. Então a família de operadores $T(t)=e^{A t}, t \geq 0$, é um semigrupo fortemente contínuo e $A$ é o gerador infinitesimal de $T(t)$.

Como o gerador infinitesimal de um $C_{0}$ semigrupo o determina unicamente, a partir daqui usaremos a notação $e^{A t}$ para indicar um $C_{0}$ semigrupo de operadores lineares limitados cujo gerador infinitesimal é $A$, mesmo que $A$ não seja limitado.

Teorema 1.1 Seja e $e^{A t}$ um $C_{0}$ semigrupo em $X$. Então:

(i) Existem constantes $M \geq 1, \omega \in \mathbb{R}$ satisfazendo:

$$
\left\|e^{A t}\right\| \leq M e^{\omega t}, \quad t \geq 0
$$

(ii) Para $x \in \mathbf{D}(A), e^{A t} x \in \mathbf{D}(A), t \rightarrow e^{A t} x$ é diferenciável $e$

$$
\frac{d}{d t} e^{A t} x=A e^{A t} x=e^{A t} A x, t>0
$$

(iii) Para $x \in D(A), t, s \in \mathbb{R}^{+}$,

$$
e^{A t} x-e^{A s} x=\int_{s}^{t} e^{A \tau} A x d \tau=\int_{s}^{t} A e^{A \tau} x d \tau
$$

(iv) $\overline{\bigcap_{n \geq 1} \mathbf{D}\left(A^{n}\right)}=X$.

Se em $(i)$ tivermos $\omega=0$, dizemos que $e^{A t}$ é uniformemente limitado. Se ainda $M=1$, então $e^{A t}$ é chamado um $C_{0}$ semigrupo de contrações .

Definição 1.3 Se $A: \mathbf{D}(A) \rightarrow X, \mathbf{D}(A) \subset X$, é um operador linear fechado, o conjunto resolvente de $A, \rho(A)$, é o conjunto de todos os números complexos $\lambda$ para os quais $\lambda I-A$ é invertivel, i.e., $R(\lambda: A)=(\lambda I-A)^{-1}$ é um operador linear limitado em $X$. $O$ espectro de $A, \sigma(A)$, é o complementar de $\rho(A)$, ou seja, $\sigma(A)=\mathbb{C} \backslash \rho(A)$. $O$ operador $R(\lambda: A)$ é chamado operador resolvente de $A$. 
Seja $X^{*}$ o espaço dual de $X$. Denotamos o valor de $x^{*} \in X^{*}$ em $x \in X$ por $\left\langle x, x^{*}\right\rangle$. Se A é um operador linear em $\mathrm{X}$, sua imagem numérica $S(A)$ é o conjunto

$$
S(A)=\left\{\left\langle A x, x^{*}\right\rangle: x \in D(A),\|x\|=1, x^{*} \in X^{*},\left\|x^{*}\right\|=1,\left\langle x, x^{*}\right\rangle=1\right\} .
$$

Teorema 1.2 Seja A um operador linear fechado densamente definido em $X$. Seja $S(A)$ a imagem numérica de $A$ e $\Sigma$ o complementar de $\overline{S(A)}$ em $\mathbb{C}$. Se $\Sigma_{0}$ é uma componente conexa de $\Sigma$ satisfazendo $\rho(A) \cap \Sigma_{0} \neq \emptyset$, então o espectro de $A$ está contido no complementar $S_{0}$ de $\Sigma_{0} e$

$$
\|R(\lambda: A)\| \leq \frac{1}{d(\lambda: \overline{S(A)})}, \quad \lambda \in \rho(A) .
$$

Para todo $x \in X$ definimos o conjunto $F(x) \subset X^{*}$ por

$$
F(x)=\left\{x^{*}: x^{*} \in X^{*} \mathrm{e}\left\langle x, x^{*}\right\rangle=\|x\|^{2}=\left\|x^{*}\right\|^{2}\right\}
$$

Segue do Teorema de Hanh-Banach que $F(x) \neq \emptyset$ para todo $x \in X$. Observamos que se $X$ é uniformemente convexo, então $F(x)$ é unitário.

Definição 1.4 Um operador linear $A$ é dissipativo se para todo $x \in \mathbf{D}(A)$ existe $u m x^{*} \in F(x)$ tal que $\operatorname{Re}\left\langle A x, x^{*}\right\rangle \leq 0$.

Teorema 1.3 (Lummer-Phillips) Seja A um operador linear com domínio denso $\mathbf{D}(A)$ em $X$.

(i) Se $A$ é dissipativo e existe um $\lambda_{0}>0$ tal que a imagem, $\mathbf{R}\left(\lambda_{0} I-A\right)$, de $\lambda_{0} I-A$ é $X$, entâo $A$ é o gerador infinitesimal de um $C_{0}$-semigrupo de contraçốes em $X$.

(ii) Se $A$ é o gerador infinitesimal de um $C_{0}$ semigrupo de contrações em $X$ então $\mathbf{R}(\lambda I-A)=X$ para todo $\lambda>0$ e $A$ é dissipativo. Além disso, para todo $x \in \mathbf{D}(A)$ e todo $x^{*} \in F(x), \operatorname{Re}\left\langle A x, x^{*}\right\rangle \leq 0$.

Definição 1.5 Seja $A$ um operador linear em $X$ satisfazendo:

- para algum $\delta, 0<\delta \leq \pi / 2$,

$$
\rho(A) \supset \Sigma_{\delta}=\left\{\lambda:|\arg \lambda|<\frac{\pi}{2}+\delta\right\}
$$

- existe $M$ tal que

$$
\|R(\lambda: A)\| \leq \frac{M}{|\lambda|}, \lambda \in \Sigma_{\delta}, \quad \lambda \neq 0 .
$$

Então dizemos que $-A$ é um operador setorial. 
Definição 1.6 Seja A um operador linear fechado, densamente definido, satisfazendo

$$
\rho(A) \supset \Sigma^{+}=\{\lambda: 0<\omega<|\arg \lambda| \leq \pi\} \cup V,
$$

onde $V$ é uma vizinhança do zero, $e$

$$
\|R(\lambda: A)\| \leq \frac{M}{1+|\lambda|}, \quad \lambda \in \Sigma^{+} .
$$

Para A satisfazendo (1.2) e (1.3) e $\alpha>0$, definimos

$$
A^{-\alpha}=\frac{1}{2 \pi i} \oint_{C} z^{-\alpha}(A-z I)^{-1} d z
$$

onde $C$ é um caminho no resolvente de $A$, de $\infty e^{-i \nu} \dot{a} \infty e^{i \nu}, \omega<\nu<\pi$, como abaixo:

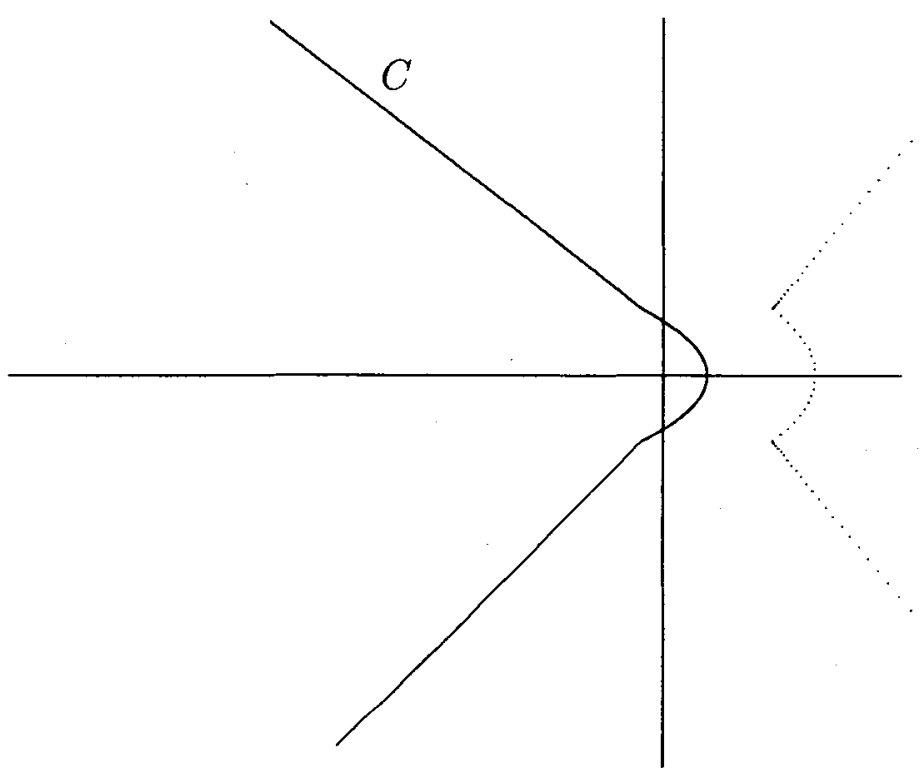

Estamos supondo que $0 \in \rho(A)$, e portanto uma vizinhança $V$ do zero pertence ao resolvente de $A$, principalmente por conveniência. A maioria dos resultados de potências fracionárias ainda valeriam mesmo que $0 \notin \rho(A)$.

Se $\alpha=n \in \mathbb{N}$, a Definição 1.6 coincide com a definição clássica de $A^{-\alpha}=A^{-n}=$ $\left(A^{n}\right)^{-1}$. Para $0<\alpha<1$ nós podemos deformar o caminho de integração no semi eixo real negativo percorrido nos dois sentidos e obter

$$
A^{-\alpha}=\frac{\sin \pi \alpha}{\pi} \int_{0}^{\infty} t^{-\alpha}(t I+A)^{-1} d t .
$$


Definição 1.7 Se $\omega<\pi / 2$, i.e., se $A$ é setorial, então $A^{-\alpha}$ é injetora $\forall \alpha>0$,e definimos

$$
A^{\alpha}:=\left(A^{-\alpha}\right)^{-1} \text {. }
$$

Teorema 1.4 As potências fracionárias do operador A satisfazem:

(i) Se $\alpha>0$, então $A^{\alpha}$ é um operador fechado;

(ii) $\alpha \geq \beta>0$ implica $\mathbf{D}\left(A^{\alpha}\right) \subset \mathbf{D}\left(A^{\beta}\right)$;

(iii) $\overline{\mathbf{D}\left(A^{\alpha}\right)}=X$ para todo $\alpha \geq 0$;

(iv) Se $\alpha, \beta$ são reais, então

$$
A^{\alpha+\beta} x=A^{\alpha} A^{\beta} x,
$$

para todo $x \in \mathbf{D}\left(A^{\gamma}\right)$, onde $\gamma=\max (\alpha, \beta, \alpha+\beta)$.

Proposição 1 Seja $H$ um espaço de Hilbert real com produto interno $\langle$,$\rangle . Seja A$ um operador auto-adjunto, $A: D(A) \subset H \rightarrow H$, satisfazendo:

(i) $\overline{D(A)}=H$;

(ii) $\langle A u, u\rangle \geq \delta\|u\|^{2}, \delta>0$.

Então $A$ é um operador setorial e $A^{\alpha}$ está bem definido, $\alpha>0$.

Prova: Como H é um espaço de Hilbert, $H$ é uniformemente convexo e o conjunto

$$
F(x)=\left\{x^{*}, x^{*} \in H^{*} \text { e }\left\langle x, x^{*}\right\rangle=\|x\|^{2}=\left\|x^{*}\right\|^{2}\right\}
$$

é unitário. Além disso, o único elemento de $F(x)$ é o funcional linear $x^{*}:=\langle., x\rangle$. Logo, a imagem numérica da $\mathrm{A}$ é

$$
S(A)=\left\{\left\langle A x, x^{*}\right\rangle=\langle A x, x\rangle,\|x\|=1\right\} .
$$

Como por hipótese $\langle A x, x\rangle \geq \delta\|x\|^{2}$, então $S(A) \subset[\delta, \infty)$.

Agora, $A$ é sobrejetor, e pela hipótese (iii) A é injetor. Com isso, $0 \in \rho(A), \mathrm{e}$ pelo Teorema $1.2, \sigma(A) \subset[\delta, \infty) \mathrm{e}$

$$
\|R(\lambda: A)\| \leq \frac{1}{d(\lambda: \overline{S(A)})}, \quad \lambda \in \rho(A) .
$$




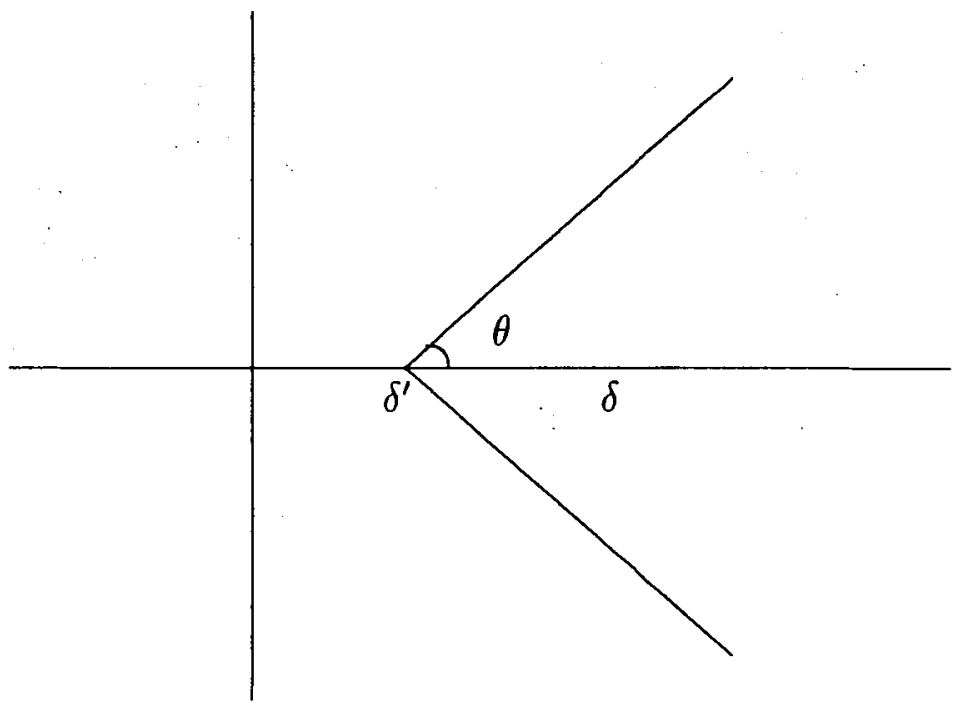

Consideremos o setor $\Sigma_{\delta^{\prime}}:=\left\{\lambda+\delta^{\prime}, \lambda \in \mathbb{C}, \theta<|\arg \lambda| \leq \pi\right\}, 0<\theta<\frac{\pi}{2}$.

É claro que $\rho(A) \supset \Sigma_{\delta^{\prime}}$ e, se $\lambda \in \Sigma_{\delta^{\prime}},(1.4)$ está satisfeita.

Se $\lambda \in \Sigma_{\delta^{\prime}}$ e $\operatorname{Re} \lambda \leq \delta$, então

$$
d(\lambda: \overline{S(A)}) \geq|\lambda-\delta|>|\lambda-\delta| \sin \theta
$$

e se $\operatorname{Re} \lambda>\delta$

$$
d(\lambda: \overline{S(A)}) \geq|\lambda-\delta| \sin \beta \geq|\lambda-\delta| \sin \theta
$$

onde $\beta$ é tal que $\theta<\beta<\pi / 2$. Portanto, $\forall \lambda \in \Sigma_{\delta^{\prime}},\|R(\lambda: A)\| \leq \frac{C_{0}}{|\lambda-\delta|}$, onde $C_{0}=\frac{1}{\sin \theta}$

Como $\frac{|\lambda|+1}{|\lambda-\delta|} \stackrel{|\lambda| \rightarrow \infty}{\longrightarrow} 1$, dado $\varepsilon>0$, existe $M$ tal que $|\lambda|>M$ implica

$$
|\lambda|+1 \leq(\varepsilon+1)|\lambda-\delta|
$$

e então existe $C_{1}$ tal que $|\lambda|>M$ implica

$$
\frac{1}{|\lambda-\delta|} \leq \frac{C_{1}}{|\lambda|+1} \quad \text { e } \quad\|R(\lambda: A)\| \leq \frac{C_{2}}{|\lambda|+1} \quad C_{2}=C_{0} C_{1} .
$$
então

Pela escolha de $\delta^{\prime}$, existe $K \neq 0$ tal que $|\lambda-\delta| \geq K, \forall \lambda \in \Sigma_{\delta^{\prime}}$. Se $|\lambda| \leq M$,

$$
|\lambda|+1 \leq M+1 \leq C_{3} K \leq C_{3}|\lambda-\delta|
$$


para alguma constante $C_{3}$. Logo

$$
\|R(\lambda: A)\| \leq \frac{C_{0}}{|\lambda-\delta|} \leq \frac{C_{4}}{|\lambda|+1}, \quad C_{4}=C_{0} C_{3} .
$$

Se $C=\max \left\{C_{2}, C_{4}\right\}$, então $\|R(\lambda: A)\| \leq \frac{C}{|\lambda|+1}$. Portanto $A$ satisfaz as condições (1.2) e (1.3) da Definição 1.6, com $\omega<\pi / 2$, e $A^{\alpha}$ está bem definido.

\subsection{Existência, Unicidade e Regularidade}

Consideremos o seguinte problema de valor inicial

$$
\left\{\begin{array}{l}
\frac{d u(t)}{d t}=A u(t)+f(t, u(t)), \quad t \geq t_{0} \\
u\left(t_{0}\right)=u_{0},
\end{array}\right.
$$

onde $A$ é o gerador infinitesimal de um $C_{0}$ semigrupo $e^{A t}$ em um espaço de Banach $X$, e $f:\left[t_{0}, T\right] \times X \rightarrow X$ é contínua em $t$ e satisfaz uma condição de Lipschitz em $u$.

Definição 1.8 Uma solução contínua da equação integral

$$
u(t)=e^{A\left(t-t_{0}\right)} u_{0}+\int_{t_{0}}^{t} e^{A(t-s)} f(s, u(s)) d s, \quad t_{0} \leq t \leq T
$$

é chamada uma solução fraca do p.v.i. (1.5). Uma função u que é quase sempre diferenciável em $\left[t_{0}, T\right]$ tal que $u^{\prime} \in L^{1}\left(t_{0}, T: X\right)$ é chamada soluçáo forte do p.v.i. (1.5) se $u\left(t_{0}\right)=u_{0}$ e $u^{\prime}(t)=A u(t)+f(t, u(t))$ quase sempre em $\left[t_{0}, T\right]$.

Suponhamos que $f$ satisfaça uma condição de Lipschitz em $u$ em subconjuntos limitados de $X$, uniformemente em $t$ em intervalos limitados, isto é, $\forall \tau \geq t_{0}$ e $c \geq 0$, existe $L(c, \tau)$ tal que

$$
\|f(t, u)-f(t, v)\| \leq L(c, \tau)\|u-v\|
$$

quaisquer que sejam $u, v \in X \operatorname{com}\|u\| \leq c,\|v\| \leq c$ e $t \in\left[t_{0}, \tau\right]$. Então temos existência e unicidade de solução fraca do p.v.i. (1.5) conforme o seguinte teorema:

Teorema 1.5 Seja $f:[0, \infty) \times X \rightarrow X$ contínua em $t$ para $t \geq 0$ e satisfazendo uma condição de Lipschitz em $u$ em subconjuntos limitados de $\bar{X}$, uniformemente 
em $t$ em intervalos limitados como em (1.6). Se A é o gerador infinitesimal de um $C_{0}$ semigrupo e $e^{A t}$ em $X$, então para todo $u_{0} \in X$ existe um $t_{\max } \leq \infty$ tal que o p.v. $i$.

$$
\left\{\begin{array}{l}
\frac{d u(t)}{d t}=A u(t)+f(t, u(t)), \quad t \geq 0 \\
u(0)=u_{0}
\end{array}\right.
$$

tem uma única solução fraca u em $\left[0, t_{m a x}\right)$. Além disso, se $t_{\max }<\infty$ então

$$
\lim _{t \uparrow t_{\max }}\|u(t)\|=\infty \text {. }
$$

Prova: Começamos mostrando que para todo $t_{0} \geq 0, u_{0} \in X$, o p.v.i.

$$
\left\{\begin{array}{l}
\frac{d u(t)}{d t}=A u(t)+f(t, u(t)), \quad t \geq t_{0} \\
u\left(t_{0}\right)=u_{0}
\end{array}\right.
$$

tem, sob as hipóteses do nosso teorema, uma única solução fraca $u$ no intervalo $\left[t_{0}, t_{1}\right]$, cujo comprimento é limitado por:

$$
\delta=\delta\left(t_{0},\left\|u_{0}\right\|\right)=\min \left\{1, \frac{\left\|u_{0}\right\|}{K\left(t_{0}\right) L\left(K\left(t_{0}\right), t_{0}+1\right)+N\left(t_{0}\right)}\right\},
$$

onde $L(c, \tau)$ é a constante de Lipschitz de $f$, como em (1.6), e

$$
\begin{aligned}
& M\left(t_{0}\right)=\max \left\{\left\|e^{A(t)}\right\|, 0 \leq t \leq t_{0}+1\right\}, \\
& K\left(t_{0}\right)=2\left\|u_{0}\right\| M\left(t_{0}\right), \\
& N\left(t_{0}\right)=\max \left\{\|f(t, 0)\|, 0 \leq t \leq t_{0}+1\right\} .
\end{aligned}
$$

Seja $t_{1}=t_{0}+\delta$. A aplicação

$$
F: \mathbf{C}\left(\left[t_{0}, t_{1}\right]: X\right) \rightarrow \mathbf{C}\left(\left[t_{0}, t_{1}\right]: X\right)
$$

definida por

$$
(F u)(t)=e^{A\left(t-t_{0}\right)} u_{0}+\int_{t_{0}}^{t} e^{A(t-s)} f(s, u(s)) d s, \quad t_{0} \leq t \leq t_{1}
$$

leva a bola de raio $K\left(t_{0}\right)$ centrada em 0 de $\mathbf{C}\left(\left[t_{0}, t_{1}\right]: X\right)$ em si mesma. Isto segue da seguinte estimativa: 


$$
\begin{aligned}
\|(F u)(t)\| & =\left\|e^{A\left(t-t_{0}\right)} u_{0}+\int_{t_{0}}^{t} e^{A(t-s)} f(s, u(s)) d s\right\| \\
& \leq M\left(t_{0}\right)\left\|u_{0}\right\|+\int_{t_{0}}^{t}\left\|e^{A(t-s)}\right\|(\|f(s, u(s))-f(s, 0)\|+\|f(s, 0)\|) d s \\
& \left.\leq M\left(t_{0}\right) \| u_{0}\right) \|+M\left(t_{0}\right) K\left(t_{0}\right) L\left(K\left(t_{0}\right), t_{0}+1\right)\left(t-t_{0}\right) \\
& +M\left(t_{0}\right) N\left(t_{0}\right)\left(t-t_{0}\right) \\
& \leq M\left(t_{0}\right)\left\|u_{0}\right\|+M\left(t_{0}\right)\left[K\left(t_{0}\right) L\left(K\left(t_{0}\right), t_{0}+1\right)+N\left(t_{0}\right)\right]\left(t-t_{0}\right) \\
& \leq M\left(t_{0}\right)\left\|u_{0}\right\| \\
& +M\left(t_{0}\right)\left[K\left(t_{0}\right) L\left(K\left(t_{0}\right), t_{0}+1\right)+N\left(t_{0}\right)\right] \frac{\left\|u_{0}\right\|}{K\left(t_{0}\right) L\left(K\left(t_{0}\right), t_{0}+1\right)+N\left(t_{0}\right)} \\
& =2 M\left(t_{0}\right)\left\|u_{0}\right\|=K\left(t_{0}\right) .
\end{aligned}
$$

Nesta bola, $f$ satisfaz a condição uniforme de Lipschitz com constante $L=L\left(K\left(t_{0}\right), t_{0}+1\right), \mathrm{e}$

$$
\|F(u)(t)-F(v)(t)\| \leq M\left(T_{0}\right) L\|u-v\|\left(t-t_{0}\right) .
$$

Com uma indução em $n$ obtemos:

$$
\left\|F^{n}(u)(t)-F^{n}(v)(t)\right\| \leq \frac{M\left(t_{0}\right) L\left(t-t_{0}\right)^{n}\|u-v\|}{n !} \leq \frac{\left(M L t_{1}\right)^{n}}{n !}\|u-v\|
$$

onde $M=M\left(t_{0}\right)$. Para $n$ suficientemente grande, $\frac{\left(M L t_{1}\right)^{n}}{n !}<1$, e pelo Teorema do Ponto Fixo de Banach, $F$ possui um único ponto fixo no intervalo $\left[t_{0}, t_{1}\right]$.

Do que acabamos de provar segue que se u é uma solução fraca de (1.7) no intervalo $[0, \tau], u$ pode ser estendida ao intervalo $[0, \tau+\delta]$ com $\delta>0$, definindo $u(t)=w(t)$ em $[\tau, \tau+\delta]$, onde $w(t)$ é a solução da equação integral:

$$
w(t)=e^{A(t-\tau)} u(\tau)+\int_{\tau}^{t} e^{A(t-s)} f(s, w(s)) d s, \quad \tau \leq t \leq \tau+\delta
$$

Além disso, $\delta$ depende apenas de $\|u(\tau)\|, K(\tau)$ e $N(\tau)$.

Seja $\left[0, t_{\max }\right)$ o intervalo maximal à direita do zero de existência da solução fraca $u$ de (1.7). Se $t_{\max }<\infty$ então

$$
\lim _{t \rightarrow t_{\max }}\|u(t)\|=\infty
$$

pois caso contrário existiria uma sequência $t_{n} \uparrow t_{\max }$ tal que $\left\|u\left(t_{n}\right)\right\| \leq C \forall n$. Isto implicaria, pelo que acabamos de provar, que para cada $t_{n}$ perto o suficiente de $t_{\max }$, 
$u$ definida em $\left[0, t_{n}\right]$ poderia ser estendida à $\left[0, t_{n}+\delta\right]$, onde $\delta>0$ é independente de $t_{n}$. Então $u$ poderia ser estendida além de $t_{\mathrm{max}}$, contrariando sua maximalidade.

Para provar a unicidade da solução fraca local $u$ de (1.7) notamos que se $v$ é uma solução fraca de (1.7) com valor inicial $v_{0}$ então

$$
\begin{aligned}
\|u(t)-v(t)\| & \leq\left\|e^{A\left(t-t_{0}\right)} u_{0}-e^{A\left(t-t_{0}\right)} v_{0}\right\|+\int_{t_{0}}^{t}\left\|e^{A(t-s)}(f(s, u(s))-f(s, v(s)))\right\| d s \\
& \leq M\left\|u_{0}-v_{0}\right\|+M L \int_{t_{0}}^{t}\|u(s)-v(s)\| d s .
\end{aligned}
$$

Pela Desigualdade de Gronwall

$$
\|u(t)-v(t)\| \leq M e^{M L\left(t-t_{0}\right)}\left\|u_{0}-v_{0}\right\| \leq M e^{M L \delta}\left\|u_{0}-v_{0}\right\| .
$$

Portanto se $u_{0}=v_{0}$, então $u=v$.

Teorema 1.6 Seja $X$ um espaço de Banach reflexivo e seja A o gerador infinitesimal de um $C_{0}$ semigrupo e em $X$. Se $f:\left[t_{0}, T\right] \rightarrow X$ é Lipschitz continua em $\left[t_{0}, T\right]$, então para todo $u_{0} \in \mathbf{D}(A)$ o p.v.i.

$$
\left\{\begin{array}{l}
\frac{d u(t)}{d t}=A u(t)+f(t), \quad t \geq t_{0} \\
u\left(t_{0}\right)=u_{0}
\end{array}\right.
$$

tem uma única solução forte em $\left[t_{0}, T\right]$ dada por

$$
u(t)=e^{A\left(t-t_{0}\right)} u_{0}+\int_{t_{0}}^{t} e^{A(t-s)} f(s) d s .
$$

Prova: Se $X$ é reflexivo e $f$ é Lipschitz contínua em $\left[t_{0}, T\right]$, então $f$ é diferenciável quase sempre, e $f^{\prime} \in L^{1}\left(t_{0}, T, X\right)$. Seja

$$
v(t)=\int_{t_{0}}^{t} e^{A(t-s)} f(s) d s, \quad t_{0} \leq t \leq T .
$$

$v(t)$ é diferenciável e $v(t) \in \mathbf{D}(A)$ quase sempre em $\left[t_{0}, T\right]$. Além disso

$$
v^{\prime}(t) \stackrel{q . s .}{=} A v(t)+f(t) \quad \in L^{1}\left(t_{0}, T, X\right) \text {. }
$$

Seja

$$
u(t)=e^{A\left(t-t_{0}\right)} u_{0}+v(t) .
$$

$\mathrm{u}(\mathrm{t})$ é diferenciável quase sempre em $\left[t_{0}, T\right] \mathrm{e}$

$$
u^{\prime}(t) \stackrel{q . s}{=} A e^{A\left(t-t_{0}\right)} u_{0}+A v(t)+f(t)=A u(t)+f(t) \quad \in L^{1}\left(t_{0}, T, X\right) .
$$

Como $u\left(t_{0}\right)=u_{0}, u(t)$ é solução forte de (1.8), e é claro que $\mathrm{u}(\mathrm{t})$ satisfaz a equação integral (1.9). 
Teorema 1.7 Seja A o gcrador infinitesimal de um $C_{0}$ semigrupo e ${ }^{A t}$ em um espaço de Banach reflexivo $X$. Se $f:\left[t_{0}, T\right] \times X \rightarrow X$ é Lipschitz contínua em ambas as variáveis, $u_{0} \in \mathbf{D}(A)$ e u é a solução fraca do problema do valor inicial (1.7), então $u$ é a solução forte deste p.v.i..

Prova: Suponhamos que $f$ satisfaça

$$
\left\|f\left(t_{1}, x_{1}\right)-f\left(t_{2}, x_{2}\right)\right\| \leq C\left(\left|t_{1}-t_{2}\right|+\left\|x_{1}-x_{2}\right\|\right), \quad t_{1}, t_{2} \in\left[t_{0}, T\right], x_{1}, x_{2} \in X .
$$

Seja $\left\|e^{A t}\right\| \leq M$ e $\|f(t, u(t))\| \leq N$ para $t_{0} \leq t \leq T$. Para $0<h<T-t_{0}$ temos

$$
\begin{aligned}
u(t+h)-u(t) & =e^{A\left(t+h-t_{0}\right)} u_{0}-e^{A\left(t-t_{0}\right)} u_{0} \\
& +\int_{t_{0}}^{t_{0}+h} e^{A(t+h-s)} f(s, u(s)) d s \\
& +\int_{t_{0}}^{t} e^{A(t-s)}[f(s+h, u(s+h))-f(s, u(s))] d s,
\end{aligned}
$$

e portanto

$$
\begin{aligned}
\|u(t+h)-u(t)\| & \leq h M\left\|A u_{0}\right\|+h M N \\
& +M C \int_{t_{0}}^{t}(h+\|u(s+h)-u(s)\|) d s \\
& \leq C_{1} h+M C \int_{t_{0}}^{t}\|u(s+h)-u(s)\| d s,
\end{aligned}
$$

que pela Desigualdade de Gronwall implica

$$
\|u(t+h)-u(t)\| \leq C_{1} e^{T M C} h
$$

Logo, $u$ é Lipschitz contínua. A continuidade Lipschitz de $u$ combinada com a continuidade Lipschitz de $f$ implica que $t \rightarrow f(t, u(t))$ é Lipschitz contínua em $\left[t_{0}, T\right]$. Do Teorema 1.6 segue que o p.v.i.

$$
\left\{\begin{array}{l}
\frac{d u(t)}{d t}=A u(t)+f(t, u(t)), \quad t \geq t_{0} \\
u\left(t_{0}\right)=u_{0}
\end{array}\right.
$$

tem uma única solução forte no intervalo $\left[t_{0}, T\right]$ satisfazendo

$$
v(t)=e^{A\left(t-t_{0}\right)} u_{0}+\int_{t_{0}}^{t} e^{A(t-s)} f(s, u(s)) d s=u(t),
$$

e então $u$ é a solução forte de (1.10). 


\subsection{Espaços de Sobolev}

Seja $\Omega$ um subconjunto aberto do $\mathbb{R}^{n}$.

Definição 1.9 Uma função $u \in L_{\text {loc }}^{1}(\Omega)$ tem $\alpha$-derivada de Sobolev se existe $v_{\alpha} \in L_{\text {loc }}^{1}(\Omega)$ tal que

$$
\int_{\Omega} \phi v_{\alpha}=(-1)^{|\alpha|} \int_{\Omega} u D^{\alpha} \phi, \quad \forall \phi \in \mathbf{C}_{c}^{\infty}(\Omega),
$$

onde $\mathbf{C}_{c}^{\infty}(\Omega)$ denota as funções com suporte compacto contido em $\Omega$ e derivadas contínuas de todas as ordens, e $\alpha=\left(\alpha_{1}, \alpha_{2}, \ldots, \alpha_{n}\right)$ é tal que $\alpha_{i} \geq 0, \alpha_{i} \in \mathbb{Z}, i=$ $1,2, \ldots, n$ e $|\alpha|=\sum \alpha_{i}$. Denotamos $D^{\alpha} u=v_{\alpha}$.

Proposição 2 A derivada de Sobolev é única e satisfaz:

(i) Se $v=D^{\alpha} u$ em $\Omega$, então $v=D^{\alpha} u$ em qualquer $\Omega^{\prime} \subset \Omega$;

(ii) Se $u_{1}$ e $u_{2}$ têm $\alpha$-derivada de Sobolev $D^{\alpha} u_{1}, D^{\alpha} u_{2}$ e $c_{1}, c_{2} \in \mathbb{G}$ entâo $c_{1} u_{1}+c_{2} u_{2}$ tem $\alpha$-derivada de Sobolev e $D^{\alpha}\left(c_{1} u_{1}+c_{2} u_{2}\right)=c_{1} D^{\alpha} u_{1}+c_{2} D^{\alpha} u_{2}$;

(iii) Se $u_{m}$ é uma sequência em $L_{l o c}^{1}(\Omega)$ tal que existe $D^{\alpha} u_{m} \in L_{l o c}^{1}(\Omega), \forall m$, e ainda

$$
\begin{aligned}
& u_{m} \stackrel{L_{\text {log }}^{1}}{\longrightarrow} u \\
& D^{\alpha} u_{m} \stackrel{\iota_{\text {log }}^{1}}{\longrightarrow} v_{\alpha}, \\
& \text { então } u \text { tem } \alpha \text {-derivada de Sobolev } D^{\alpha} u=v_{\alpha} .
\end{aligned}
$$

Definição 1.10 Seja $m \in \mathbb{Z}, m \geq 0$ e $\Omega \subset \mathbb{R}^{n}$ aberto, e seja $p$ tal que $1 \leq p \leq \infty$. Definimos os Espaços de Sobolev $\bar{W}^{m, p}(\Omega)$ da seguinte forma:

$$
W^{m, p}(\Omega):=\left\{u \in L^{p}(\Omega) \text { tal que } \exists D^{\alpha} u \in L^{p}(\Omega), \forall 0 \leq|\alpha| \leq m\right\} .
$$

$W^{m, p}(\Omega)$ é um espaço de Banach com a norma \|\|$_{m, p}$ dada por

$$
\|u\|_{m, p}=\left(\sum_{0 \leq|\alpha| \leq m}\left\|D^{\alpha} u\right\|_{L^{p}}^{p}\right)^{1 / p}
$$

onde

$$
\begin{gathered}
\|u\|_{L^{p}}=\left(\int_{\Omega}|u|^{p}\right)^{1 / p}, \quad 1 \leq p<\infty, \epsilon \\
\|u\|_{L^{\infty}}=\inf \{k \geq 0:|u(x)| \leq k \text { quase sempre }\} .
\end{gathered}
$$

Equivalentemente, $W^{m, p}(\Omega)$ é o completamento de $\left\{u \in \mathbf{C}^{m}(\Omega):\|u\|_{m, p}<\infty\right\}$ com relação à norma \|\|$_{m, p}$. $W_{0}^{m, p}(\Omega)$ é o fecho de $\mathbf{C}_{c}^{\infty}(\Omega)$ em $W^{m, p}(\Omega)$. 
$W^{m, p}(\Omega)$ é separável se $1 \leq p<\infty$, reflexivo e uniformemente convexo se $1<p<\infty$.

$W^{m, 2}(\Omega)$ é um espaço de Hilbert com produto interno

$$
\langle u, v\rangle_{m}=\sum_{0 \leq|\alpha| \leq m}\left\langle D^{\alpha} u, D^{\alpha} v\right\rangle
$$

onde $\langle$,$\rangle é o produto interno em L^{2}(\Omega)$. Usamos a notação $H^{m}(\Omega)=W^{m, 2}(\Omega)$ e $H_{0}^{m}(\Omega)=W_{0}^{m, 2}(\Omega)$.

Teorema 1.8 (partições da unidade) - Seja $\Gamma$ um compacto do $\mathbb{R}^{n}$ e sejam $U_{1}, U_{2}, \ldots, U_{k}$ abertos satisfazendo $\Gamma \subset \bigcup_{i=1}^{k} U_{i}$. Existem funções $\theta_{0}, \theta_{1}, \ldots, \theta_{k} \in$ $\mathbf{C}^{\infty}\left(\mathbb{R}^{n}\right)$ de tal forma que

(i) $0 \leq \theta_{i} \leq 1, \quad \forall i=1,2, \ldots, k e \sum_{i=0}^{k} \theta_{i}=1$;

(ii) $\theta_{i}$ tem suporte compacto, supp $\theta_{i} \subset U_{i}, \forall i=1,2, \ldots, k$, e supp $\theta_{0} \subset \mathbb{R}^{n} \backslash \Gamma$.

Se $\Omega$ é um aberto limitado e $\Gamma=\partial \Omega$, então $\left.\theta_{0}\right|_{\Omega} \in \mathbf{C}_{c}^{\infty}(\Omega)$.

Teorema 1.9 Seja $u \in L^{p}(\Omega)$ com $1<p \leq \infty$. As seguintes propriedades são equivalentes:

(i) $u \in W^{1, p}(\Omega)$;

(ii) Existe uma constante C tal que

$$
\left|\int_{\Omega} u \frac{\partial \varphi}{\partial x_{i}}\right| \leq C\|\varphi\|_{L^{p^{\prime}}} \quad \forall \varphi \in \mathbf{C}_{c}^{\infty}(\Omega), \quad \forall i=1,2, \ldots, n,
$$

onde $p^{\prime}$ é tal que

$$
\frac{1}{p}+\frac{1}{p^{\prime}}=1
$$

(iii) Existe uma constante $C$ tal que para todo aberto $\omega \subset \subset \Omega$ e todo $h \in \mathbb{R}^{n}$ com $|h|<d\left(\omega, \mathbb{R}^{n} \backslash \Omega\right)$ tem-se

$$
\left\|\tau_{h} u-u\right\|_{L^{p}(\omega)} \leq C|h|
$$

onde $\tau_{h} u(x)=u(x+h)$. Além disso a constante $C$ pode ser tomada como $C=\|\nabla u\|_{L^{p}}$ em (ii) e (iii). 
Definição 1.11 Dizemos que um aberto $\Omega$ é de classe $\mathbf{C}^{m}, m \in \mathbb{Z}, m \geq 1$, se para todo $x \in \partial \Omega$ existe uma vizinhança $U$ de $x$ em $\mathbb{R}^{n}$ e uma aplicação bijetora $H: Q \rightarrow U$ tal que

- $H \in \mathbf{C}^{m}(\bar{Q})$

- $H^{-1} \in \mathbf{C}^{m}(\bar{U})$

- $H\left(Q_{+}\right)=U \cap \Omega$

- $H\left(Q_{0}\right)=U \cap \partial \Omega$

onde

$$
\begin{aligned}
Q & =\left\{x=\left(x^{\prime}, x_{n}\right) \in \mathbb{R}^{n-1} \times \mathbb{R} ;\left|x^{\prime}\right|<1 \text { e }\left|x_{n}\right|<1\right\} \\
Q_{+} & =Q \cap \mathbb{R}_{+}^{n} \\
Q_{0} & =\left\{x \in Q ; x_{n}=0\right\}
\end{aligned}
$$

Teorema 1.10 Seja $\Omega \subset \mathbb{R}^{3}, \Omega$ aberto de classe $\mathbf{C}^{1}$ com fronteira limitada. Então

$$
H^{1}(\Omega) \subset L^{p}(\Omega), \quad 1 \leq p \leq 6,
$$

e a imersão é contínua. Se $1 \leq p<6$, a imersão é compacta.

Teorema 1.11 Seja $\Omega$ um aberto de classe $\mathbf{C}^{2}$ com $\partial \Omega$ limitada ou $\Omega=\mathbb{R}_{+}^{n}$. Seja $f \in L^{2}(\Omega)$ e seja $u \in H_{0}^{1}(\Omega)$ satisfazendo:

$$
\int_{\Omega} \nabla u \nabla \varphi=\int_{\Omega} f \varphi, \quad \forall \varphi \in H_{0}^{1}(\Omega)
$$

Então $u \in H^{2}(\Omega)$ e $\|u\|_{H^{2}} \leq C\|f\|_{L^{2}}$, onde $C$ é uma constante que só depende de $\Omega$.

\section{Prova:}

Caso a: $\Omega=\mathbb{R}^{n}$.

Dado $h \in \mathbb{R}^{n}, h \neq 0$ seja

$$
D_{h} u=\frac{1}{|h|}\left(\tau_{h} u-u\right), \quad \text { i.e., } \quad D_{h} u(x)=\frac{u(x+h)-u(x)}{|h|},
$$


e seja $\varphi=D_{-h}\left(D_{h} u\right) . \varphi \in H_{0}^{1}\left(\mathbb{R}^{n}\right)$ e por $(1.11)$

$$
\int_{\Omega}\left|\nabla D_{h} u\right|^{2}=\int_{\Omega} \nabla u \nabla \varphi=\int_{\Omega} f \varphi=\int_{\Omega} f D_{-h}\left(D_{h} u\right),
$$

portanto,

$$
\left\|D_{h} u\right\|_{H^{1}}^{2} \leq\|f\|_{L^{2}}\left\|D_{-h}\left(D_{h} u\right)\right\|_{L^{2}} .
$$

Pelo Teorema 1.9 temos

$$
\left\|D_{-h} u\right\|_{L^{2}\left(\mathbf{R}^{n}\right)} \leq\|\nabla u\|_{L^{2}\left(\mathbf{R}^{n}\right)} .
$$

Logo

$$
\left\|D_{h} u\right\|_{H^{1}}^{2} \leq\|f\|_{L^{2}}\left\|\nabla D_{h} u\right\|_{L^{2}}=\|f\|_{L^{2}}\left\|D_{h} u\right\|_{H^{1}}
$$

e então

$$
\left\|D_{h} u\right\|_{H^{1}} \leq\|f\|_{L^{2}}
$$

Em particular,

$$
\left\|D_{h} \frac{\partial u}{\partial x_{j}}\right\|_{L^{2}} \leq\|f\|_{L^{2}} \quad j=1,2, \ldots, n .
$$

Pelo Teorema $1.9 \frac{\partial u}{\partial x_{j}} \in H^{1}(\Omega), j=1,2, \ldots, n$, e então $u \in H^{2}(\Omega)$. É claro que $\exists C$ tal que $\|u\|_{H^{2}} \leq C\|f\|_{L^{2}}$.

Caso b: $\Omega=\mathbb{R}_{+}^{n}$.

Seja $h \in \mathbb{R}^{n-1} \times\{0\}$. Dizemos que $h$ é paralelo à fronteira de $\Omega$ e denotamos $h \| \partial \Omega$. Se $u \in H_{0}^{1}(\Omega)$ e $h \| \partial \Omega$, então $\tau_{h} u \in H_{0}^{1}(\Omega)$. Seja $\varphi=D_{-h}\left(D_{h} u\right)$. Como no caso a:

$$
\left\|D_{h} u\right\|_{H^{1}}^{2} \leq\|f\|_{L^{2}}\left\|D_{-h}\left(D_{h} u\right)\right\|_{L^{2}}
$$

Se $h \| \partial \Omega$ ainda temos

$$
\left\|D_{h} v\right\|_{L^{2}} \leq\|\nabla v\|_{L^{2}} \quad \forall v \in H^{1}(\Omega) .
$$

Portanto

$$
\left\|D_{h} u\right\|_{H^{1}} \leq\|f\|_{L^{2}} \quad \forall h \| \partial \Omega
$$

Sejam $1 \leq j \leq n, 1 \leq k \leq n-1, h=(0,0, \ldots, \overbrace{|h|}^{k \underline{a}}, 0, \ldots, 0)$ e $\varphi \in \mathbf{C}_{c}^{\infty}(\Omega)$. Temos

$$
\int D_{h}\left(\frac{\partial u}{\partial x_{j}}\right) \varphi=-\int u D_{-h}\left(\frac{\partial \varphi}{\partial x_{j}}\right)
$$

e por (1.12)

$$
\left|\int u D_{-h}\left(\frac{\partial \varphi}{\partial x_{j}}\right)\right| \leq\|f\|_{L^{2}}\|\varphi\|_{L^{2}}
$$


Passando o limite quando $|h| \rightarrow 0$ obtemos

$$
\left|\int u \frac{\partial^{2} \varphi}{\partial x_{j} \partial x_{k}}\right| \leq\|f\|_{L^{2}}\|\varphi\|_{L^{2}}, \quad 1 \leq j \leq n, \quad 1 \leq k \leq n-1 .
$$

Falta mostrarmos que

$$
\left|\int u \frac{\partial^{2} \varphi}{\partial x_{n}^{2}}\right| \leq C\|f\|_{L^{2}}\|\varphi\|_{L^{2}} \quad \forall \varphi \in \mathbf{C}_{c}^{\infty}(\Omega)
$$

Notemos que a equação (1.11) implica em

$$
\left|\int u \frac{\partial^{2} \varphi}{\partial x_{n}^{2}}\right| \leq \sum_{i=1}^{n-1}\left|\int u \frac{\partial^{2} \varphi}{\partial x_{i}^{2}}\right|+\left|\int f \varphi\right| \leq C\|f\|_{L^{2}}\|\varphi\|_{L^{2}}, \forall \varphi \in \mathbf{C}_{c}^{\infty}(\Omega) .
$$

Logo temos

$$
\left|\int u \frac{\partial^{2} \varphi}{\partial x_{j} \partial x_{k}}\right| \leq C\|f\|_{L^{2}}\|\varphi\|_{L^{2}} \quad \forall \varphi \in \mathbf{C}_{c}^{\infty}(\Omega), \quad 1 \leq j, k \leq n .
$$

Então pelo Teorema $1.9, \frac{\partial u}{\partial x_{j}} \in H^{1}(\Omega), j=1,2, \ldots, n$, e portanto $u \in H^{2}(\Omega)$.

Caso geral: $\Omega$ limitado.

Seja $U_{1}, U_{2}, \ldots, U_{k}$ uma cobertura finita de $\partial \Omega$ e consideremos uma partição da unidade $\theta_{1}, \theta_{2}, \ldots, \theta_{k}$, subordinada a esta cobertura conforme o Teorema 1.8. É claro que $u=\sum_{i=0}^{k} \theta_{i} u$, e assim basta mostrarmos que $\theta_{i} u \in H^{2}(\Omega), i=0,1, \ldots, k$. Primeiro demonstramos que $\theta_{0} u \in H^{2}(\Omega)$. Como $\left.\theta_{0} u\right|_{\Omega} \in \mathbf{C}_{c}^{\infty}(\Omega)$, a função $\theta_{0} u$ estendida por 0 fora de $\Omega$ pertence a $H^{1}\left(\mathbb{R}^{n}\right)$. Segue da equação (1.11) que $\theta_{0} u$ satisfaz

$$
\int_{\mathbf{R}^{n}} \nabla\left(\theta_{0} u\right) \nabla \varphi=\int_{\mathbf{R}^{n}} \theta_{0} f \varphi-\int_{\mathbf{R}^{n}} 2 \nabla \theta_{0} \nabla u \varphi-\int_{\mathbf{R}^{n}}\left(\triangle \theta_{0}\right) u \varphi, \quad \forall \varphi \in H_{0}^{1}\left(\mathbb{R}^{n}\right) .
$$

Se $g=\theta_{0} f-2 \nabla \theta_{0} \nabla u-\left(\triangle \theta_{0}\right) u$, então $g \in L^{2}\left(\mathbb{R}^{n}\right)$, e segue portanto do caso a que $\theta_{0} u \in H^{2}\left(\mathbb{R}^{n}\right)$ com

$$
\left\|\theta_{0} u\right\|_{H^{2}} \leq C\left(\|f\|_{L^{2}}+\|u\|_{H^{1}}\right) \leq C^{\prime}\|f\|_{L^{2}},
$$

já que $\|u\|_{H^{1}} \leq\|f\|_{L^{2}}$, pela equação (1.11).

Por fim demonstramos que $\theta_{i} u \in H^{2}(\Omega)$ para $1 \leq i \leq k$. Seja $v=\theta_{i} u \in$ $H_{0}^{1}\left(\Omega \cap U_{i}\right)$. Como acima, $v$ satisfaz

$$
\int \nabla v \nabla \varphi=\int g \varphi, \forall \varphi \in H_{0}^{1}\left(\Omega \cap U_{i}\right)
$$


onde

$$
g=\theta_{i} f-2\left(\nabla \theta_{i}\right)(\nabla u)-\left(\triangle \theta_{i}\right) u
$$

e $g \in L^{2}\left(\Omega \cap U_{i}\right)$ com $\|g\|_{L^{2}} \leq C\|f\|_{L^{2}}$. Se $H$ é como na Definição 1.11 e $J=H^{-1}$, seja $\omega(y)=v(H(y)), y \in Q_{+}$e $\omega(J x)=v(x), x \in \Omega \cap U_{i}$. Seja $\psi \in H_{0}^{1}\left(Q_{+}\right)$e seja $\varphi(x)=\psi(J x), x \in \Omega \cap U_{i}$. Então $\varphi \in H_{0}^{1}\left(\Omega \cap U_{i}\right) \mathrm{e}$

$$
\begin{gathered}
\frac{\partial v}{\partial x_{j}}=\sum_{k} \frac{\partial \omega}{\partial y_{k}} \frac{\partial J_{k}}{\partial x_{j}} \\
\frac{\partial \varphi}{\partial x_{j}}=\sum_{\ell} \frac{\partial \psi}{\partial y_{\ell}} \frac{\partial J_{\ell}}{\partial x_{j}} .
\end{gathered}
$$

Logo

$$
\begin{aligned}
\int_{\Omega \cap U_{i}} \nabla v \nabla \varphi d x & =\int_{\Omega \cap U_{i}, k, \ell} \sum_{j} \frac{\partial J_{k}}{\partial x_{j}} \frac{\partial J_{\ell}}{\partial x_{j}} \frac{\partial \omega}{\partial y_{k}} \frac{\partial \psi}{\partial y_{\ell}} d x \\
& =\int_{Q_{+}} \sum_{j, k, \ell} \frac{\partial J_{k}}{\partial x_{j}} \frac{\partial J_{\ell}}{\partial x_{j}} \frac{\partial \omega}{\partial y_{k}} \frac{\partial \psi}{\partial y_{\ell}}|J a c H| d y .
\end{aligned}
$$

Portanto

$$
\int_{\Omega \cap U_{i}} \nabla v \nabla \varphi=\int_{Q_{+}} \sum_{k, \ell} a_{k \ell} \frac{\partial \omega}{\partial y_{k}} \frac{\partial \psi}{\partial y_{\ell}} d y
$$

onde

$$
a_{k \ell}=\sum_{j} \frac{\partial J_{k}}{\partial x_{j}} \frac{\partial J_{\ell}}{\partial x_{j}}|J a c H| .
$$

Notemos que $a_{k \ell} \in \mathbf{C}^{1}\left(\overline{Q_{+}}\right)$e que qualquer que seja $\xi \in \mathbb{R}^{n}$, temos

$$
\sum_{k, \ell} a_{k \ell} \xi_{k} \xi_{\ell}=|J a c H| \sum_{j}\left|\sum_{k} \frac{\partial J_{k}}{\partial x_{j}} \xi_{k}\right|^{2} \geq \alpha|\xi|^{2}
$$

com $\alpha>0$, uma vez que os jacobianos Jac $H$ e Jac $J$ são não singulares.

Por outro lado, temos

$$
\int_{\Omega \cap U_{i}} g \varphi d x=\int_{Q_{+}}(g \circ H) \psi|J a c H| d y
$$

Portanto temos

$$
\sum_{k, \ell=1}^{n} \int_{Q_{+}} \boldsymbol{a}_{k, \ell} \frac{\partial \omega}{\partial y_{k}} \frac{\partial \psi}{\partial y_{\ell}} d y=\int_{Q_{+}} \tilde{g} \psi d y \quad \forall \psi \in H_{0}^{1}\left(Q_{+}\right)
$$

onde $\tilde{g}=(g \circ H)|J a c H| \in L^{2}\left(Q_{+}\right)$. 
Mostraremos que $\omega \in H^{2}\left(Q_{+}\right)$e que $\|\omega\|_{H^{2}} \leq C\|\tilde{g}\|_{L^{2}}$. Seja $\psi=D_{-h}\left(D_{h} \omega\right)$ com $h \| Q_{0}$ e $|h|$ pequeno o suficiente para que $\psi \in \bar{H}_{0}^{1}\left(Q_{+}\right)$. Assim,

$$
\sum_{k, \ell} \int_{Q_{+}} D_{h}\left(a_{k \ell} \frac{\partial \omega}{\partial y_{k}}\right) \frac{\partial}{\partial y_{\ell}}\left(D_{h} \omega\right)=\int_{Q_{+}} \tilde{g} D_{-h}\left(D_{h} \omega\right)
$$

Mas

$$
\int_{Q_{+}} \tilde{g} D_{-h}\left(D_{h} \omega\right) \leq\|\tilde{g}\|_{L^{2}}\left\|D_{-h}\left(D_{h} \omega\right)\right\|_{L^{2}} \leq\|\tilde{g}\|_{L^{2}}\left\|\nabla D_{h} \omega\right\|_{L^{2}}
$$

Por outro lado

$$
D_{h}\left(a_{k \ell} \frac{\partial \omega}{\partial y_{k}}\right)(y)=a_{k \ell}(y+h) \frac{\partial}{\partial y_{k}} D_{h} \omega(y)+\left(D_{h} a_{k \ell}(y)\right) \frac{\partial \omega}{\partial y_{k}}(y),
$$

e portanto temos

$$
\sum_{k, \ell} \int_{Q_{+}} D_{h}\left(a_{k \ell} \frac{\partial \omega}{\partial y_{k}}\right) \frac{\partial}{\partial y_{\ell}}\left(D_{h} \omega\right) \geq \alpha\left\|\nabla D_{h} \omega\right\|_{L^{2}}^{2}-\|\omega\|_{H^{1}}\left\|\nabla D_{h} \omega\right\|_{L^{2}}
$$

Assim obtemos

$$
\left\|\nabla D_{h} \omega\right\|_{L^{2}} \leq C_{1}\left(\|\omega\|_{H^{1}}+\|\tilde{g}\|_{L^{2}}\right) \leq C_{2}\|\tilde{g}\|_{L^{2}} .
$$

Pela equação 1.13 e pela desiguadade de Poincaré temos $\|\omega\|_{L^{1}} \leq C_{3}\|\tilde{g}\|_{L^{2}}$. Concluímos como no caso b que

$$
\left|\int_{Q_{+}} \frac{\partial \omega}{\partial y_{k}} \frac{\partial \psi}{\partial y_{\ell}}\right| \leq C\|\tilde{g}\|_{L^{2}}\|\psi\|_{L^{2}} \quad \forall \psi \in \mathbf{C}_{c}^{1}\left(Q_{+}\right), \quad \forall(k, \ell) \neq(n, n)
$$

Para concluirmos que $\omega \in H^{2}\left(Q_{+}\right)$e $\|\omega\|_{H^{2}} \leq C\|\tilde{g}\|_{L^{2}}$, falta demonstrarmos que

$$
\left|\int_{Q_{+}} \frac{\partial \omega}{\partial y_{n}} \frac{\partial \psi}{\partial y_{n}}\right| \leq C\|\tilde{g}\|_{L^{2}}\|\psi\|_{L^{2}} \quad \forall \psi \in \mathbf{C}_{c}^{1}\left(Q_{+}\right)
$$

Se substituirmos, na equação (1.13), $\phi$ por $\frac{1}{a_{n n}} \phi, \phi \in \mathbf{C}_{c}^{1}\left(Q_{+}\right)$, teremos

$$
\int a_{n n} \frac{\partial \omega}{\partial y_{n}} \frac{\partial}{\partial y_{n}}\left(\frac{1}{a_{n n}} \psi\right)=\int \frac{\tilde{g}}{a_{n n}} \psi-\sum_{(k, \ell) \neq(n, n)} \int a_{k \ell} \frac{\partial \omega}{\partial y_{k}} \frac{\partial}{\partial y_{\ell}}\left(\frac{1}{a_{n n}} \psi\right),
$$

quer dizer:

$$
\begin{aligned}
\int \frac{\partial \omega}{\partial y_{n}} \frac{\partial \psi}{\partial y_{n}} & =\int \frac{1}{a_{n n}}\left(\frac{\partial a_{n n}}{\partial y_{n}}\right) \frac{\partial \omega}{\partial y_{n}} \psi+\int \frac{\tilde{g}}{a_{n n}} \psi \\
& +\sum_{(k, \ell) \neq(n, n)} \int \frac{\partial \omega}{\partial y_{k}}\left(\frac{\partial a_{k \ell}}{\partial y_{\ell}}\right) \frac{\psi}{a_{n n}}-\sum_{(k, \ell) \neq(n, n)} \int \frac{\partial \omega}{\partial y_{k}} \frac{\partial}{\partial y_{\ell}}\left(\frac{a_{k \ell}}{a_{n n}} \psi\right) .
\end{aligned}
$$


Combinando (1.14) e (1.15) obtemos

$$
\left|\int_{Q_{+}} \frac{\partial \omega}{\partial y_{n}} \frac{\partial \psi}{\partial y_{n}}\right| \leq C\left(\|\omega\|_{H^{1}}+\|\tilde{g}\|_{L^{2}}\right)\|\psi\|_{L^{2}} \quad \forall \psi \in \mathbf{C}_{c}^{1}\left(Q_{+}\right) .
$$

Portanto $\omega \in H^{2}\left(Q_{+}\right)$e $\|\psi\|_{H^{2}} \leq C\|\tilde{g}\|_{L^{2}}$. Voltando a $\Omega \cap U_{i}$, temos que $v=$ $\theta_{i} u \in H^{2}\left(\Omega \cap U_{i}\right)$ e então $\theta_{i} u \in H^{2}(\bar{\Omega}) \operatorname{com}\left\|\theta_{i} u\right\|_{H^{2}} \leq C\|f\|_{L^{2}}$.

Teorema 1.12 (Lax-Milgran) Seja $H$ um espaço de Hilbert, e seja a(u,v) uma forma bilinear, contínua e coerciva. Então para todo $\varphi \in H^{*}$ existe um único $u \in H$ tal que

$$
a(u, v)=\langle v, \varphi\rangle \quad \forall v \in H .
$$

Além disso, se a é simétrica, então u se caracteriza por:

$$
u \in H e \frac{1}{2} a(u, u)-\langle\varphi, u\rangle=\min _{v \in H}\left\{\frac{1}{2} a(v, v)-\langle\varphi, v\rangle\right\}
$$

Teorema $1.13 \overline{\left\{u \in \mathbf{C}^{2}(\bar{\Omega}): u=0 \text { em } \partial \Omega\right\}}{ }^{H^{2}}=H_{0}^{1}(\Omega) \cap H^{2}(\Omega)$.

Teorema 1.14 Seja $\Omega$ um subconjunto aberto, limitado e com fronteira suave do $\mathbb{R}^{3}$. Seja $\mathcal{A}$ o operador

$$
\begin{gathered}
\mathcal{A}: \mathbf{D}(\mathcal{A}) \subset X \rightarrow X \\
(\mathcal{A} u) x=-(\triangle u) x=-\left(\frac{\partial^{2} u}{\partial x_{1}{ }^{2}}+\frac{\partial^{2} u}{\partial x_{2}{ }^{2}}+\frac{\partial^{2} u}{\partial x_{3}{ }^{2}}\right) x, \quad x \in \Omega
\end{gathered}
$$

onde $X=L^{2}(\Omega)$ e $\mathbf{D}(\mathcal{A})=H^{2}(\Omega) \cap H_{0}^{1}(\Omega)$. Então:

(i) $\mathcal{A}$ é fechado;

(ii) $\mathcal{A}$ é simétrico;

(iii) $\mathcal{A}$ é sobrejetor;

(iv) $\mathcal{A}$ é positivo;

(v) $\mathcal{A}$ tem inversa compacta.

\section{Prova:}


(i) $\mathcal{A} u=-\triangle u=-\left(D^{\alpha_{1}} u+D^{\alpha_{2}} u+D^{\alpha_{3}} u\right)$, onde

$$
\begin{aligned}
& \alpha_{1}=(2,0,0) \\
& \alpha_{2}=(0,2,0) \\
& \alpha_{3}=(0,0,2)
\end{aligned}
$$

Se $\left\{u_{m}\right\} \in \mathbf{D}(\mathcal{A})=H^{2}(\Omega) \cap H_{0}^{1}(\Omega)$,

$$
u_{m} \stackrel{L^{2}}{\longrightarrow} u \Rightarrow u_{m} \stackrel{L_{\text {log }}}{\longrightarrow} u
$$

$\mathrm{e} \exists\left\{D^{\alpha_{i}} u_{m}\right\}, i=1,2,3$,

$$
D^{\alpha_{i}} u_{m} \stackrel{L^{2}}{\longrightarrow} v_{i} \Rightarrow D^{\alpha_{i}} u_{m} \stackrel{L_{\text {log }}^{1}}{\longrightarrow} v_{i}
$$

então $v_{i}=D^{\alpha_{i}} u_{i}, i=1,2,3$. Ou seja, se $u_{m} \stackrel{L^{2}}{\longrightarrow} u$ e $\mathcal{A} u_{m} \stackrel{L^{2}}{\longrightarrow} u$ então $v=\mathcal{A} u$.

(ii) Se $u, v \in \mathbf{D}(\mathcal{A})$, então pelo Teorema 1.13 existe uma sequência $\left\{v_{n}\right\} \subset \mathbf{C}^{2}(\bar{\Omega})$, $v_{n}=0$ em $\partial \Omega$ tal que $v_{n} \stackrel{H^{2}}{\longrightarrow} v, \operatorname{logo}$

$$
\begin{aligned}
\langle\mathcal{A} u, v\rangle & =\left\langle\mathcal{A} u, \lim v_{n}\right\rangle=\lim \left\langle\mathcal{A} u, v_{n}\right\rangle \\
& =\lim \left(-\int_{\Omega} \triangle u v_{n}\right)=\lim \int_{\Omega} \nabla u \nabla v_{n} \\
& =\lim \left(-\int_{\Omega} u \triangle v_{n}\right)=\lim \left\langle u, \mathcal{A} v_{n}\right\rangle \\
& =\left\langle u, \lim \mathcal{A} v_{n}\right\rangle=\langle u, \mathcal{A} v\rangle,
\end{aligned}
$$

onde $\langle$,$\rangle é o produto interno em L^{2}(\Omega)$.

(iii) $H_{0}^{1}(\Omega)=W_{0}^{1,2}(\Omega)={\overline{\mathbf{C}_{c}^{\infty}(\Omega)}}^{W^{1,2}(\Omega)}$ é um espaço de Hilbert com o produto interno

$$
\langle u, v\rangle_{1,2}=\sum_{0 \leq|\alpha| \leq 1}\left\langle D^{\alpha} u, D^{\alpha} v\right\rangle \text {. }
$$

Seja $a(u, v)=\int_{\Omega} \nabla u(x) \nabla v(x) d x, u, v \in H_{0^{1}}(\Omega)$.

(a) $a(u, v)$ é bilinear:

$$
\begin{aligned}
& \begin{aligned}
a\left(u_{1}+u_{2}, v\right) & =\int_{\Omega} \nabla\left(u_{1}+u_{2}\right)(x) \nabla v(x) d x \\
& =\int_{\Omega} \nabla u_{1}(x) \nabla v(x) d x+\int_{\Omega} \nabla u_{2}(x) \nabla v(x) d x \\
& =a\left(u_{1}, v\right)+a\left(u_{2}, v\right) .
\end{aligned} \\
& \text { Analogamente } a\left(u, v_{1}+v_{2}\right)=a\left(u, v_{1}\right)+a\left(u, v_{2}\right) .
\end{aligned}
$$


(b) $a(u, v)$ é contínua:

$$
\|a(u, v)\|=\left|\int_{\Omega} \nabla u(x) \nabla v(x) d x\right| \leq\|\nabla u\|_{L^{2}}\|\nabla v\|_{L^{2}} \leq\|u\|_{H^{1}}\|v\|_{H^{1}} .
$$

(c) $a(u, v)$ é coerciva:

$a(v, v)=\int_{\Omega} \nabla v(x) \nabla v(x) d x=\|\nabla v\|_{2}^{2} \geq C\|v\|_{2}^{2}$, pela desigualdade de Poincaré.

Assim, dada $h \in L^{2}(\Omega)$, seja $L_{h}: H_{0}^{1}(\Omega) \rightarrow \mathbb{R}$, dada por

$$
L_{h}(v)=\int_{\Omega} h(x) v(x) d x, \quad \forall v \in H_{0}^{1}(\Omega) .
$$

Pelo teorema de Lax-Milgran existe uma única $u \in H_{0}^{1}(\Omega)$ tal que

$$
a(u, v)=L_{h}(v) \quad \forall v \in H_{0}^{1}(\Omega),
$$

ou seja

$$
\int_{\Omega} \nabla u(x) \nabla v(x) d x=\int_{\Omega} h(x) v(x) d x \quad \forall v \in H_{0}^{1}(\Omega) .
$$

Agora, como demonstramos no Teorema 1.11, $u \in H^{2}(\Omega)$, logo $u \in \mathbf{D}(\mathcal{A})$, então:

$$
\int_{\Omega} h(x) v(x) d x=\int_{\Omega} \nabla u(x) \nabla v(x) d x=-\int_{\Omega} \triangle u(x) v(x) d x \quad \forall v \in H_{0}^{1}(\Omega) .
$$

Portanto $h=-\triangle u=\mathcal{A} u$. Logo $\mathcal{A}$ é sobrejetora.

(iv) $\langle\mathcal{A} u, u\rangle=-\int_{\Omega} \Delta u u=\int_{\Omega} \nabla u \nabla u=\|\nabla u\|_{L^{2}}^{2} \geq C\|u\|_{L^{2}}^{2}$,

pela desigualdade de Poincaré.

(v) Se \|\|$_{G}$ denota a norma do gráfico em $\mathbf{D}(\mathcal{A})$, então

$$
\mathcal{A}:\left(\mathbf{D}(\mathcal{A}),\|\|_{G}\right) \longrightarrow L^{2}(\Omega)
$$

é uma isometria. Logo, se $B$ é um subconjunto limitado de $L^{2}(\Omega), \mathcal{A}^{-1}(B)$ é um subconjunto limitado de $\left(\mathbf{D}(\mathcal{A}),\|\|_{G}\right)$. Como $\left(\mathbf{D}(\mathcal{A}),\|\|_{G}\right)$ está compactamente imerso em $L^{2}(\Omega)$, então $\mathcal{A}^{-1}$ é compacta.

Corolário 10 operador $\mathcal{A}$

$$
\begin{gathered}
\mathcal{A}: \mathbf{D}(\mathcal{A}) \subset X \rightarrow X \\
(\mathcal{A} u) x=-(\triangle u) x=-\left(\frac{\partial^{2} u}{\partial x_{1}{ }^{2}}+\frac{\partial^{2} u}{\partial x_{2}{ }^{2}}+\frac{\partial^{2} u}{\partial x_{3}{ }^{2}}\right) x, \quad x \in \Omega,
\end{gathered}
$$

onde $X=L^{2}(\Omega)$ e $\mathbf{D}(\mathcal{A})=H^{2}(\Omega) \cap H_{0}^{1}(\Omega)$ é auto-adjunto. 
Combinando a Proposição 1 e o Teorema 1.14 temos que $\mathcal{A}^{\alpha}$ está bem definida $\forall \alpha>0$.

Mostremos que $\mathbf{D}\left(\mathcal{A}^{1 / 2}\right)=X^{1 / 2}=H_{0}^{1}(\Omega)$. Como $L^{2}(\Omega)$ é um espaço de Hilbert separável e $\mathcal{A}^{-1}$ é um operador compacto e auto-adjunto, então $L^{2}(\Omega)$ admite uma base Hilbertiana formada por auto vetores de $\mathcal{A}$. Ou seja $\forall u \in L^{2}(\Omega)$,

$$
u=\sum_{i=1}^{\infty}\left\langle u, \phi_{n}\right\rangle \phi_{n}
$$

onde $\phi_{n}$ é auto vetor de $\mathcal{A}$ qualquer que seja $n$. Se $u \in \mathbf{D}(\mathcal{A})$,

$$
\mathcal{A} u=\sum_{i=1}^{\infty} \lambda_{n}\left\langle u, \phi_{n}\right\rangle \phi_{n}
$$

onde $\lambda_{n}$ é auto valor de $\mathcal{A}$ qualquer que seja $n$.

Cada $\phi_{n} \in \mathbf{D}(\mathcal{A})$ e então

$$
\int_{\Omega} \nabla \phi_{n} \nabla \phi_{m}=-\int_{\Omega} \Delta \phi_{n} \phi_{m}=\int_{\Omega} \mathcal{A} \phi_{n} \phi_{m}=\lambda_{n} \int_{\Omega} \phi_{n} \phi_{m}=0, \quad m \neq n,
$$

e $\left\{\phi_{n}\right\}$ é uma sequência ortogonal em $H^{1}(\Omega)$. De forma análoga mostra-se que $\left\|\nabla \phi_{n}\right\|_{L^{2}}=\lambda_{n}^{1 / 2}$. Disto segue que, se \|\|$_{1 / 2}$ denota a norma do gráfico em $X^{1 / 2}$, então

$$
\|u\|_{H^{1}}^{2}=\left\|\sum_{i=1}^{\infty}\left\langle u, \phi_{n}\right\rangle \nabla \phi_{n}\right\|_{L^{2}}^{2}=\sum_{i=1}^{\infty}\left|\left\langle u, \phi_{n}\right\rangle\right|^{2} \lambda_{n}=\|u\|_{1 / 2}^{2} .
$$

Portanto

$$
H_{0}^{1}(\Omega)={\overline{\mathbf{C}_{c}^{\infty}(\Omega)}}^{H^{1}(\Omega)} \subset{\overline{H^{2}(\Omega) \cap H_{0}^{1}(\Omega)}}^{H^{1}(\Omega)}={\overline{H^{2}(\Omega) \cap H_{0}^{1}(\Omega)}}^{x^{1 / 2}}=X^{1 / 2} .
$$

Por outro lado, se $u \in X^{1 / 2}$, e $\phi_{n}, \lambda_{n}$ são auto vetores e auto valores de $\mathcal{A}$ respectivamente, $n \geq 1$,

$$
u=\sum_{n=1}^{\infty}\left\langle u, \phi_{n}\right\rangle \phi_{n} \quad \text { implica } \quad \mathcal{A}^{1 / 2} u=\sum_{n=1}^{\infty} \lambda_{n}^{1 / 2}\left\langle u, \phi_{n}\right\rangle \phi_{n} .
$$

De fato,

$$
(\mathcal{A}-\lambda I) u=\left(\begin{array}{cccc}
\lambda_{1}-\lambda & 0 & 0 & \ldots \\
0 & \lambda_{2}-\lambda & 0 & \ldots \\
\vdots & \ddots & \vdots & \vdots
\end{array}\right)\left(\begin{array}{c}
\left\langle u, \phi_{1}\right\rangle \\
\left\langle u, \phi_{2}\right\rangle \\
\vdots
\end{array}\right)
$$


então

$$
(\mathcal{A}-\lambda I)^{-1} u=\left(\begin{array}{cccc}
\left(\lambda_{1}-\lambda\right)^{-1} & 0 & 0 & \ldots \\
0 & \left(\lambda_{2}-\lambda\right)^{-1} & 0 & \ldots \\
\vdots & \vdots & \ddots & \vdots
\end{array}\right)\left(\begin{array}{c}
\left\langle u, \phi_{1}\right\rangle \\
\left\langle u, \phi_{2}\right\rangle \\
\vdots
\end{array}\right)
$$

Logo,

$$
(\mathcal{A}-\lambda I)^{-1} \phi_{n}=\left(\lambda_{n}-\lambda\right)^{-1} \phi_{n} .
$$

Agora, o caminho $C$ na definição de $A^{-\alpha}$, se $\alpha<1$, pode ser tomado como a reunião de círculos $C_{n}$ em torno dos auto valores $\lambda_{n}, n \geq 1$.

Então

$$
\begin{aligned}
\mathcal{A}^{-\alpha} \phi_{n} & =\frac{1}{2 \pi i} \oint_{C} z^{-\alpha}(\mathcal{A}-z I)^{-1} d z \phi_{n} \\
& =\frac{1}{2 \pi i} \sum_{m} \oint_{C_{m}} z^{-\alpha}(\mathcal{A}-z I)^{-1} d z \phi_{n} \\
& =\frac{1}{2 \pi i} \sum_{m} \oint_{C_{m}} z^{-\alpha}\left(\lambda_{n}-z I\right)^{-1} \phi_{n} d z \\
& =\frac{1}{2 \pi i} \oint_{C_{n}} z^{-\alpha}\left(\lambda_{n}-z I\right)^{-1} \phi_{n} d z \\
& =\lambda_{n}^{-\alpha} \phi_{n} .
\end{aligned}
$$

Logo,

$$
\mathcal{A}^{-\alpha} u=\left(\begin{array}{cccc}
\lambda_{1}^{-\alpha} & 0 & 0 & \cdots \\
0 & \lambda_{2}^{-\alpha} & 0 & \ldots \\
\vdots & \vdots & \ddots & \vdots
\end{array}\right)\left(\begin{array}{c}
\left\langle u, \phi_{1}\right\rangle \\
\left\langle u, \phi_{2}\right\rangle \\
\vdots
\end{array}\right)
$$

Seja $u_{k}=\sum_{n=1}^{k}\left\langle u, \phi_{n}\right\rangle \phi_{n}, u_{k} \in H_{0}^{1}(\Omega) \cap H^{2}(\Omega)$. Então 


$$
\begin{aligned}
\left\|u-u_{k}\right\|_{1 / 2} & =\left\|\mathcal{A}^{1 / 2}\left(u-u_{k}\right)\right\|_{L^{2}}=\left\|\sum_{n=1}^{\infty} \lambda_{n}^{1 / 2}\left\langle u-u_{k}, \phi_{n}\right\rangle \phi_{n}\right\|_{L^{2}} \\
& =\left\|\sum_{n=1}^{\infty} \lambda_{n}^{1 / 2}\left\langle u, \phi_{n}\right\rangle \phi_{n}-\sum_{n=1}^{\infty} \lambda_{n}^{1 / 2}\left\langle\sum_{j=1}^{k}\left\langle u, \phi_{j}\right\rangle \phi_{j}, \phi_{n}\right\rangle \phi_{n}\right\|_{L^{2}} \stackrel{k \rightarrow \infty}{\longrightarrow} 0 . \\
\text { Portanto } & \left\|u-u_{k}\right\|_{H^{1}}=\left\|u-u_{k}\right\|_{X^{1 / 2}} \longrightarrow 0 .
\end{aligned}
$$

Como $H_{0}^{1}(\Omega)$ é fechado, $u \in H_{0}^{1}(\Omega)$. Portanto, $X^{1 / 2} \subset H_{0}^{1}(\Omega)$. 


\section{Capítulo 2}

\section{Atratores para semigrupos e equações de evolução}

O objetivo deste capítulo é estudar o comportamento assintótico de semigrupos (em geral não lineares). Mais especificamente, o objetivo principal é dar condições suficientes sob as quais um semigrupo possui um atrator global minimal. Os resultados apresentados neste capítulo seguem Ladyzhenskaya [1991] e serão utilizados no capítulo seguinte para mostrar a existência de atratores globais para o semigrupo gerado pela equação das ondas com atrito linear e termo forçante com crescimento subcrítico.

Por todo este capítulo, $X$ denota um espaço métrico completo.

Definição 2.1 Um semigrupo $T(t), t \in \mathbb{R}^{+}$, é uma família de operadores contínuos $T(t): X \rightarrow X$ satisfazendo a seguinte propriedade:

$$
T\left(t_{1}\right) T\left(t_{2}\right) x=T\left(t_{1}+t_{2}\right) x, \quad \forall t_{1}, t_{2} \in \mathbb{R}^{+} \text {e } x \in X .
$$

Dizemos que o semigrupo $T(t)$ é pontualmente contínuo se para cada $x \in X$ a aplicação $t \rightarrow T(t) x$ de $\mathbb{R}^{+} \rightarrow X$ é contínua. Se a aplicação $(t, x) \rightarrow T(t) x$ de $\mathbb{R}^{+} \times X \rightarrow X$ é contínua, dizemos que o semigrupo $T(t)$ é contínuo.

Dado um semigrupo $T(t)$ e $A \subset X$ usaremos a seguinte notação :

$$
\begin{gathered}
\gamma^{+}(A)=\bigcup_{x \in A}\left\{T(t) x, t \in R^{+}\right\} \\
\gamma_{\left[t_{1}, t_{2}\right]}^{+}(A)=\bigcup_{x \in A}\left\{T(t) x, t \in\left[t_{1}, t_{2}\right]\right\} ; \\
\gamma_{t}^{+}(A)=\bigcup_{x \in A}\{T(\tau) x, \tau \in[t, \infty)\} .
\end{gathered}
$$


Dizemos que $\gamma^{+}(A)$ é a semi-órbita positiva de $A$. Em particular se $A=\{x\}$, dizemos que $\gamma^{+}(x)=\gamma^{+}(\{x\})$ é a semi-órbita positiva de $\mathrm{x}$.

O semigrupo $T(t)$ é limitado se para cada subconjunto limitado $B \subset X, \gamma^{+}(B)$ é limitado.

Definição 2.2 Sejam $A$ e $M$ subconjuntos de $X$. Dizemos que $A$ atrai $M$ se dado $\varepsilon>0$ existe $t_{1}>0, t_{1}$ dependendo de $\varepsilon$ e $M$, tal que $T(t)(M) \subset \mathcal{O}_{\varepsilon}(A) \forall t>t_{1}$, onde $\mathcal{O}_{\varepsilon}(A)=\cup_{x \in A} B_{\varepsilon}(x)$, e $B_{\varepsilon}(x)$ é a bola de centro $x$ e raio $\varepsilon . A \subset X$ atrai $o$ ponto $x$ se $A$ atrai $\{x\}$.

Definição 2.3 Se $A \subset X$, o conjunto w-limite $\omega(A)$ é definido por

$$
\omega(A)=\left\{y \in X: y=\lim _{k \rightarrow \infty} T\left(t_{k}\right) x_{k}, x_{k} \in A, t_{k} \rightarrow \infty\right\} .
$$

Se $x \in X$, definimos o conjunto $\omega$-limite $\omega(x):=\omega(\{x\})$.

Os conjuntos $\omega$-limites podem ser descritos em termos de $\gamma_{t}^{+}(x)=\gamma_{t}^{+}(\{x\}) \mathrm{e}$ $\gamma_{t}^{+}(A)$ como mostra o lema a seguir:

Lema 2.1 Se para cada $B \subset X, \bar{B}$ denota o fecho de $B$ em $X$, temos:

$$
\begin{aligned}
& \omega(x)=\bigcap_{t \geq 0} \overline{\gamma_{t}^{+}(x)} ; \\
& \omega(A)=\bigcap_{t \geq 0} \overline{\gamma_{t}^{+}(A)},
\end{aligned}
$$

Prova: Se $y \in \omega(A)$ então

$$
y=\lim _{k \rightarrow \infty} T\left(t_{k}\right) x_{k}, \quad x_{k} \in A \text { e } t_{k} \rightarrow \infty
$$

Assim, para todo $t \geq 0$ existe $k_{0}$ tal que se $k \geq k_{0}, t_{k} \geq t$ e $\left\{T\left(t_{k}\right) x_{k}\right\}_{k \geq k_{0}}$ é uma sequência em $\gamma_{t}^{+}(A)$ convergindo para y. Portanto $y \in \bigcap_{t \geq 0} \overline{\gamma_{t}^{+}(A)}$.

Por outro lado, se $y \in \bigcap_{t>0} \overline{\gamma_{t}^{+}(A)}$, dado $k \in \mathbb{N}$, existe $y_{k} \in \gamma_{k}^{+}(A)$ tal que $d\left(y, y_{k}\right)<k^{-1}$. Como $y_{k} \in \gamma_{k}^{+}(A), y_{k}=T\left(t_{k}\right) x_{k}$, com $t_{k} \geq k$ e $x_{k} \in A$. Logo $T\left(t_{k}\right) x_{k} \rightarrow y$ quando $k \rightarrow \infty$ e $y \in \omega(A)$.

Definição 2.4 Um semigrupo $T(t)$ pertence à classe $\mathcal{K}$ se para cada $t>0$ o operador $T(t)$ é compacto, i.e., dado um conjunto limitado $B \subset X, T(t)(B)$ é précompacto. 
Teorema 2.1 Seja $T(t)$ um semigrupo de classe $K$. Seja $A \subset X$ e suponhamos que exista $\tau \in \mathbb{R}^{+}$tal que $\gamma_{\tau}^{+}(A)$ é limitado. Então:

(i) $\omega(A)$ é não vazio e compacto;

(ii) $\omega(A)$ atrai $A$;

(iii) $\omega(A)$ é invariante, i. e., $T(t)(\omega(A)) \doteq \omega(A), \forall t \in \mathbb{R}^{+}$;

(iv) $\omega(A)$ é o menor conjunto fechado que atrai $A$;

(v) Se $T(t)$ é contínuo e $A$ é conexo então $\omega(A)$ é conexo.

\section{Prova:}

(i) $T(t)\left(\gamma_{\tau}^{+}(A)\right)=\gamma_{t_{+\tau}}^{+}(A)$ é pré-compacto se $t>0$, e $\gamma_{t_{2}+\tau}^{+}(A) \subset \gamma_{t_{1}+\tau}^{+}(A)$ para todo $t_{2}>t_{1}$. Logo

$$
\omega(A)=\bigcap_{t>0} \overline{\gamma_{t}^{+}(A)}=\bigcap_{t>0} \overline{\gamma_{t+\tau}^{+}(A)}
$$

é a intersecção de uma família decrescente de conjuntos compactos. Portanto $\omega(A)$ é não vazio e compacto.

(ii) Suponhamos que $\omega(A)$ não atraia $A$. Então existe $\varepsilon>0$ e uma sequência $t_{k} \rightarrow \infty, t_{k} \geq \tau+1$ tal que $T\left(t_{k}\right)(A) \not \subset \mathcal{O}_{\varepsilon}(\omega(A))$, i.e., existe $x_{k} \in A$ tal que $T\left(t_{k}\right) x_{k} \notin \mathcal{O}_{\varepsilon}(\omega(A))$. Logo $\left\{T\left(t_{k}\right) x_{k}\right\} \subset T(\tau+1)(A)=T(1)\left(\gamma_{\tau}^{+}(A)\right) \mathrm{e}$

$$
d\left(T\left(t_{k}\right) x_{k}, \omega(A)\right) \geq \varepsilon \quad \forall k .
$$

Como $T(1)\left(\gamma_{\tau}^{+}(A)\right)$ é pré-compacto, é possível extrair da sequência acima uma subsequência convergente $\left\{T\left(t_{k_{j}}\right) x_{k_{j}}\right\}, k_{j} \stackrel{j \rightarrow \infty}{\longrightarrow} \infty$. Seja $y=\lim _{j \rightarrow \infty} T\left(t_{k_{j}}\right) x_{k_{j}}$. Então $y \in \omega(A)$, contradizendo $(2.1)$.

(iii) Se $x \in \omega(A), x=\lim _{k \rightarrow \infty} T\left(t_{k}\right) x_{k}, x_{k} \in A$ e $t_{k} \rightarrow \infty$, então dado $t \in \mathbb{R}^{+}$,

$$
T(t) x=T(t)\left(\lim _{k \rightarrow \infty} T\left(t_{k}\right) x_{k}\right)=\lim _{k \rightarrow \infty} T\left(t+t_{k}\right) x_{k} .
$$

Logo, $T(t) x \in \omega(A)$. Além disso, podemos assumir $1+\tau+t \leq t_{1}<t_{2}<\ldots$ Os pontos $y_{k}=T\left(t_{k}-t\right) x_{k}, k=1,2, \ldots$, pertencem ao conjunto pré-compacto $\gamma_{\tau+1}^{+}(A)$. Então a sequência $\left\{y_{k}\right\}$ admite uma subsequência convergente $\left\{y_{k_{j}}\right\}$ e $\lim _{j \rightarrow \infty} y_{k_{j}}=y \in \omega(A)$. Portanto

$$
x=\lim _{j \rightarrow \infty} T\left(t_{k_{j}}\right) x_{k_{j}}=\lim _{j \rightarrow \infty} T(t) y_{k_{j}}=T(t) y \in T(t)(\omega(A)) .
$$


(iv) Suponhamos que exista $F$ fechado, propriamente contido em $\omega(A)$ e atraindo $A$. $F$ é compacto desde que $\omega(A)$ é. Seja $y \in \omega(A) \backslash F$ e seja $\varepsilon>0$ pequeno o suficiente para que

$$
\mathcal{O}_{\varepsilon}(y) \cap \mathcal{O}_{\varepsilon}(F)=\emptyset \text {. }
$$

Existe $t_{\varepsilon}>0$ tal que $T(t)(A) \subset \mathcal{O}_{\varepsilon}(F), t \geq t_{\varepsilon}$. Mas $y=\lim _{k \rightarrow \infty} T\left(t_{k}\right) x_{k}$, $x_{k} \in A$ e $t_{k} \rightarrow \infty$. Então $T\left(t_{k}\right)(A) \cap \mathcal{O}_{\varepsilon}(y) \neq \emptyset$ se $t_{k}$ for suficientemente grande, o que contradiz (2.2).

(v) Sejam $F_{1}$ e $F_{2}$ fechados satisfazendo $F_{1} \cap F_{2}=\emptyset$ e $\omega(A)=F_{1} \cup F_{2}$. Existe $\varepsilon>0$ tal que $\overline{\mathcal{O}_{\varepsilon}\left(F_{1}\right)} \cap \overline{\mathcal{O}_{\varepsilon}\left(F_{2}\right)}=\emptyset$, e é claro que $\mathcal{O}_{\varepsilon}(\omega(A))=\mathcal{O}_{\varepsilon}\left(F_{1}\right) \cup \mathcal{O}_{\varepsilon}\left(F_{2}\right)$. Como $\omega(A)$ atrai $A$, existe $t_{1}=t_{1}(\varepsilon, A)$ tal que $\gamma_{t}^{+}(A) \subset \mathcal{O}_{\varepsilon}(\omega(A)), t \geq t_{1}$. Agora, como supomos $T(t)$ contínuo e A conexo, então $\gamma_{t}^{+}(A)$ é conexo. Logo, para todo $t \geq t_{1}$, ou $\gamma_{t}^{+}(A) \subset \mathcal{O}_{\varepsilon}\left(F_{1}\right)$ ou $\gamma_{t}^{+}(A) \subset \mathcal{O}_{\varepsilon}\left(F_{2}\right)$. Portanto ou $\omega(A)=\bigcap_{t \geq t_{1}} \overline{\gamma_{t}^{+}(A)} \subset F_{1}$, ou $\omega(A) \subset F_{2}$, e assim, $F_{1}=\emptyset$ ou $F_{2}=\emptyset$.

Definição 2.5 Uma órbita completa $\gamma(x)$ de um ponto $x \in X$ é uma curva $x(t)$, $-\infty<t<+\infty$, satisfazendo:

- $x(t) \in X \forall t \in \mathbb{R}$;

- $x(0)=x$;

- $T(\tau) x(t)=x(t+\tau) \forall t \in \mathbb{R} e \tau \in \mathbb{R}^{+}$.

Dizemos que $\gamma^{-}(x)=\{x(t),-\infty<t \leq 0\}$ é uma semi-órbita negativa de $x$. Assim, $\gamma(x)=\gamma^{+}(x) \cup \gamma^{-}(x)$.

Em geral, para $x \in X$ arbitrário, a órbita completa $\gamma(x)$ pode não existir, ou mesmo quando existe, pode não ser única. Porém, se $A$ é um subconjunto invariante de $X$ e os operadores $T(t)$ são invertiveis em $A$, então temos unicidade da órbita $\gamma(x)$ para cada $x \in A$ conforme o lema abaixo.

Lema 2.2 Seja $A$ um subconjunto invariante de $X$. Então para todo $x \in A$ existe uma órbita completa $\gamma(x)$. Se o semigrupo $T(t)$ é pontualmente contínuo, a órbita $\gamma(x)$ é uma curva contínua em $A$. Se os operadores $T(t), t \in \mathbb{R}^{+}$, são invertiveis em $A$, então:

(i) por cada $x \in A$ passa uma única órbita $\gamma(x)$;

(ii) a familia de operadores $\{T(t), t \in \mathbb{R}\}$, onde $T(t):=(T(-t))^{-1}$ se $t<0$, satisfaz a propriedade de grupo $T\left(t_{1}+t_{2}\right)=T\left(t_{1}\right) T\left(t_{2}\right), \forall t_{1}, t_{2} \in \mathbb{R}$. Se A for compacto, então $\{T(t), t \in \mathbb{R}\}$ é um grupo de operadores contínuos. Este grupo é pontualmente contínuo se $\left\{T(t), t \in \mathbb{R}^{+}\right\}$for pontualmente contínuo, é contínuo se $\left\{T(t), t \in \mathbb{R}^{+}\right\}$for contínuo. 
Desde que $T(1)(A)=A$, dado $x \in A$ existe uma sequência $\left\{x_{-k}\right\}_{k=1}^{\infty}$ de pontos $\operatorname{de} A$ satisfazendo $T(1) x_{-1}=x$ e $T(1) x_{-k-1}=x_{-k}, k>0$. Unimos os pontos $x_{-k-1}$ e $x_{-k}$ pela curva $\left\{T(t) x_{-k-1}, t \in[0,1]\right\}$. A coleção de todas estas curvas forma $\gamma^{-}(x)$ e $x(t):=T(t+k+1) x_{-k-1}, t \in[-k-1,-k], k=0,1, \ldots$

Segue da construção de $\gamma^{-}(x)$ que, se o semigrupo $\mathrm{T}(\mathrm{t})$ for pontualmente contínuo então $\gamma(x)=\gamma^{+}(x) \cup \gamma^{-}(x)$ é contínua.

As afirmações do lema seguem então da construção da órbita $\gamma(x)$ e da definição de $T(t), t<0$.

Definição 2.6 Dizemos que um subconjunto $A$ de $X$ é um atrator de pontos global (para o semigrupo $T(t)$ ) se $A$ atrai cada ponto $x$ de $X$. Se $A$ atrai cada conjunto limitado. $B \subset X$, entẫo $A$ é um atrator global.

Definição 2.7 Se existir um atrator de pontos global limitado para o semigrupo $T(t)$, então dizemos que $T(t)$ é pontualmente dissipativo. Se existir um atrator global limitado, dizemos que $T(t)$ é $B$-dissipativo.

Definição $2.8 U m$ conjunto $A \subset X$ é chamado absorvente se para todo $x \in X$ existe $t_{1}(x) \in \mathbb{R}^{+}$tal que $T(t) x \in A$ para todo $t \geq t_{1}$. A é $B$-absorvente se para todo conjunto limitado $B \subset X$ existe $t_{1}(B) \in \mathbb{R}^{+}$, tal que $T(t)(B) \subset A$ para todo $t \geq t_{1}(B)$.

Teorema 2.2 Seja $T(t)$ um semigrupo de classe $\mathcal{K}$. Suponhamos que $T(t)$ ou é $B$-dissipativo ou é pontualmente dissipativo e limitado. Então $T(t)$ tem um atrator global minimal $\mathcal{M}$, que é compacto e invariante. Além disso $\mathcal{M}$ é conexo se $X$ for conexo.

Prova: Se $T(t)$ é $B$-dissipativo e $B_{0}$ é seu atrator global limitado, então dado $\varepsilon_{0}>0$, $B_{1}:=\mathcal{O}_{\varepsilon_{0}}\left(B_{0}\right)$ é um conjunto $B$-absorvente global limitado. Neste caso

$$
\mathcal{M}=\omega\left(B_{1}\right)=\omega\left(\mathcal{O}_{\varepsilon_{0}}\left(B_{0}\right)\right) \text {. }
$$

De fato, para todo conjunto limitado $B \subset X$ existe $t_{B} \geq 0$ tal que $T(t)(B) \subset B_{1}$ se $t \geq t_{B}$. Em particular, $T(t)\left(B_{1}\right) \subset B_{1}$ se $t \geq t_{B_{1}}$. Logo, $\gamma_{t_{B_{1}}}^{+}\left(B_{1}\right) \subset B_{1}$ é limitado. Pelo Teorema 2.1, $\omega\left(B_{1}\right)$ é um conjunto não vazio, compacto e invariante, e $\omega\left(B_{1}\right)$ atrai $B_{1}$. Portanto, para todo $\varepsilon>0$ existe $t_{1}(\varepsilon) \geq 0$ tal que $T(t)\left(B_{1}\right) \subset \mathcal{O}_{\varepsilon}\left(\omega\left(B_{1}\right)\right)$, $t \geq t_{1}(\varepsilon)$, e então, dado qualquer conjunto limitado $B \subset X$,

$$
T(t)(B) \subset \mathcal{O}_{\varepsilon}\left(\omega\left(B_{1}\right)\right), \forall t \geq t_{1}(\varepsilon)+t_{B} .
$$

Portanto $\omega\left(B_{1}\right)$ é um atrator global fechado. A minimalidade segue do fato que $\omega\left(B_{1}\right)$ é o menor conjunto fechado que atrai $B_{1}$.

Suponhamos agora que $T(t)$ é um semigrupo limitado e pontualmente dissipativo. Seja $B_{2}$ um atrator de pontos global limitado. Escolhemos $\varepsilon_{2}>0$ e definimos $B_{3}$ 
$:=\mathcal{O}_{\varepsilon_{2}}\left(B_{2}\right)$ e $B_{4}:=\gamma^{+}\left(B_{3}\right)$. Então $\mathcal{M}=\omega\left(B_{3}\right)$. De fato, desde que $B_{2}$ é um atrator de pontos global, para todo ponto $x \in X$ existe $t_{x} \geq 0$ tal que $T\left(t_{x}\right) x \in B_{3}$. Como $B_{3}$ é um conjunto aberto e o operador $T\left(t_{x}\right)$ é contínuo, segue que $T\left(t_{x}\right)\left(\mathcal{O}_{\varepsilon_{x}} x\right) \subset B_{3}$ para algum $\varepsilon_{x}>0$. Então

$$
T\left(t_{x}+t\right)\left(\mathcal{O}_{\varepsilon_{x}} x\right) \subset T(t)\left(B_{3}\right) \subset B_{4} \quad \forall t \geq 0 .
$$

Agora, dado $K$ compacto, seja $\left\{\mathcal{O}_{\varepsilon_{x_{i}}}\left(x_{i}\right)\right\}_{i=1,2, \ldots, r}, x_{1}, x_{2}, \ldots, x_{r} \in K$ uma cobertura finita de $K$. Seja $\varepsilon_{K}$ tal que $\mathcal{O}_{\varepsilon_{K}}(K) \subset \cup_{i=1}^{r} \mathcal{O}_{\epsilon_{x_{i}}}\left(x_{i}\right)$ e $t_{K}=\max _{1 \leq i \leq r}\left\{t_{x_{i}}\right\} \geq 0$. Então

$$
T(t)\left(\mathcal{O}_{\varepsilon_{K}}(K)\right) \subset B_{4} \quad \forall t \geq t_{K}
$$

Como $T(t)$ é limitado, segue do teorema 1 que todo conjunto limitado $B$ é atraído por $\omega(B)$. Então $T(t)(B) \subset \mathcal{O}_{\varepsilon_{1}}(\omega(B)), t \geq t_{1}\left(\varepsilon_{1}, B\right), \forall \varepsilon_{1}>0$. Desde que $\omega(B)$ é compacto, podemos escolher $\varepsilon_{1}=\varepsilon_{\omega(B)}$ e segue de (2.3) que

$$
T\left(t+t_{1}\right)(B) \subset B_{4}, t \geq t_{w(B)} \text { e } t_{1}=t_{1}\left(\varepsilon_{1}, B\right) .
$$

Portanto $B_{4}$ é um $B$-absorvente limitado global, e como demonstramos acima, $\omega\left(B_{4}\right)=\mathcal{M}$. Mas pelas definições de $B_{3}$ e $B_{4}, T(t)\left(B_{4}\right)=\gamma_{t}^{+}\left(B_{3}\right)$. Também

$$
\gamma_{t}^{+}\left(B_{4}\right)=\gamma_{t}^{+}\left(\gamma^{+}\left(B_{3}\right)\right)=\bigcup_{s \geq t} T(s)\left(\gamma^{+}\left(B_{3}\right)\right)=\gamma_{t}^{+}\left(B_{3}\right) .
$$

Logo $\omega\left(B_{4}\right)=\omega\left(B_{3}\right)$. A minimalidade de $\omega\left(B_{3}\right)$ segue do Teorema 2.1. Finalmente, se existe um conexo $B \supset \mathcal{M}$, então $\mathcal{M}$ é conexo, desde que $T(t)(B)$ é conexo qualquer que seja $t \in \mathbb{R}^{+}$e, para $\varepsilon>0$,

$$
\mathcal{M}=T(t)(\mathcal{M}) \subset T(t)(B) \subset \mathcal{O}_{\varepsilon}(\mathcal{M}), t \geq t_{1}(\varepsilon, B) .
$$

Como um corolário da prova do teorema anterior obtemos o seguinte resultado:

Proposição 3 Se o semigrupo $T(t)$ é limitado e pontualmente dissipativo então existe um conjunto limitado $B_{0}$ tal que para todo compacto $K$

$$
T(t)\left(\mathcal{O}_{e_{K}}(K)\right) \subset B_{0} \quad \forall t \geq t_{K}
$$

com algum $\varepsilon_{K}>0$ e $t_{K} \geq 0$. Além disso $T(t)\left(B_{0}\right) \subset B_{0}, \quad \forall t \in \mathbb{R}^{+}$.

Proposição 4 Se $T(t)$ é um semigrupo de classe $\mathcal{K}$ limitado e pontualmente dissipativo ou $B$-dissipativo, então o atrator minimal $\mathcal{M}$ pode ser caracterizado como segue:

(i) $\mathcal{M}=\bigcup_{B \in \mathcal{B}} \omega(B)$, onde $\mathcal{B}$ é a coleção de todos os subconjuntos limitados de $X$;

(ii) $\mathcal{M}_{X}=\bigcup_{K \in \mathcal{K}} \omega(K)$, onde $\mathcal{K}$ é a coleção de todos os subconjuntos compactos em $X$ 
(iii) $\mathcal{M}$ é a união de todas as órbitas completas limitadas em $X$;

(iv) $\mathcal{M}$ é a união de todas as órbitas completas pré-compactas em $X$;

(v) $\mathcal{M}$ é o conjunto invariante maximal em $X$;

(vi) $\widehat{\mathcal{M}}=\overline{\bigcup_{x \in X} \omega(x)}$, onde $\widehat{\mathcal{M}}$ denota o atrator de pontos global fechado minimal.

Prova:

(i) Todo conjunto limitado $B$ é atraído para seu conjunto $\omega$-limite $\omega(B)$. Isso é claro se $T(t)$ é limitado e, se $T(t)$ é $B$-dissipativo com atrator global limitado $A$, então

$$
\gamma_{\tau}^{+}(B) \subset \mathcal{O}_{\varepsilon}(A) \in \mathcal{B}
$$

se $\tau$ for grande o suficiente. Agora, $\omega(B)$ é invariante e $\mathcal{M}$ atrai $\omega(B)$, então $\omega(B) \subset \mathcal{O}_{\varepsilon}(\mathcal{M}), \forall \varepsilon>0$. Como $\omega(B)$ é fechado, $\omega(B) \subset \mathcal{M}$, e desde que $\mathcal{M}$ é invariante e compacto, $\omega(\mathcal{M})=\mathcal{M}$. Então $\mathcal{M}=\omega(\mathcal{M}) \subset \bigcup_{B \in \mathcal{B}} \omega(B) \subset \mathcal{M}$.

(ii) $\bigcup_{K \in \mathcal{K}} \omega(K) \subset \bigcup_{B \in \mathcal{B}} \omega(B)=\mathcal{M}=\omega(\mathcal{M}) \subset \bigcup_{K \in \mathcal{K}} \omega(K)$.

(iii) e (iv) Pelo lema 2.2, por cada ponto $x \in \mathcal{M}$ passa uma órbita completa $\gamma(x)$. Qualquer tal órbita está em $\mathcal{M}$ e então é limitada e pré-compacta. Portanto $\mathcal{M}$ está contido na reunião de todas as órbitas completas limitadas em $X$, e $\mathcal{M}$ está contido na reunão de todas as órbitas completas pré-compactas em $X$. Por outro lado, Seja $\gamma(x)=\{x(t), t \in \mathbb{R}\}$ uma órbita completa limitada passando por algum $x=x_{0} \in X$. Desde que $\gamma(x)$ é invariante e limitada e o semigrupo $T(t)$ é de classe $\mathcal{K}, \gamma(x)$ é pré-compacta. Logo, $B:=\overline{\gamma(x)}$ é um conjunto compacto invariante. Portanto $\omega(B)=B$ e então $B=\omega(B) \subset \mathcal{M}=\bigcup_{B \in \mathcal{B}} \omega(B)$.

(v) Se B é um conjunto invariante limitado, então $T(t)(B)=B$ e portanto $\omega(B)=$ $B$ e $B \subset \mathcal{M}$. Uma vez que $\mathcal{M}$ é um conjunto invariante limitado $\mathcal{M}$ é o maior conjunto invariante limitado.

(vi) $\forall x \in X, \omega(x) \subset \widehat{\mathcal{M}}$, pois $\omega(x)$ é o menor fechado que atrai $x$. Por outro lado, $\widehat{\bigcup_{x \in X} \omega(x)}$ é um atrator global fechado que contém $\widehat{\mathcal{M}}$ pelo caráter minimal de $\widehat{\mathcal{M}}$.

Definição 2.9 Uma função de Liapunov é uma função contínua $\mathcal{L}: X \rightarrow \mathbb{R}$ estritamente decrescente ao longo de cada $\gamma^{+}(x)$, exceto em pontos estacionários $z, T(t) z=z, \forall t \in \mathbb{R}^{+}$. 
Teorema 2.3 Suponhamos que o semigrupo $T(t)$ pertence à classe $\mathcal{K}$ e $\gamma^{+}(x)$ é limitado para todo $x \in X$. Se para este semigrupo existe uma função de Liapunov $\mathcal{L}$, entâo seu atrator de pontos minimal global coincide com o conjunto $\mathcal{Z}=\{z$ : $T(t) z=z, \forall t \geq 0\}$. Se $\mathcal{Z}$ for um conjunto limitado e $T(t)$ for limitado, então o semigrupo possui um atrator global minimal $\mathcal{M}$ compacto e invariante. Se $X$ for conexo, então $\mathcal{M}$ é. Ambos os extremos de qualquer órbita completa $\gamma(x) \subset$ $\mathcal{M}$ tendem para $\mathcal{Z}$ quando $t \rightarrow \pm \infty$ correspondentemente. Se $X$ é conexo e $\mathcal{Z}$ não, então $\mathcal{Z}$ está propriamente contido em $\mathcal{M}$ e o atrator $\mathcal{M}$ consiste das órbitas completas que conectam pontos de $\mathcal{Z}$.

Prova: Se $T(t)$ é de classe $\mathcal{K}$ e $\gamma^{+}(x)$ é limitado para todo $x \in X$, então qualquer que seja $x \in X$ o limite $\lim _{t \rightarrow \infty} \mathcal{L}(T(t) x) \equiv \ell_{+}(x)$ existe e $\mathcal{L}$ restrita à $\omega(x)$ é constante e igual à $\ell_{+}(x)$, pois se $y \in \omega(x), y=\lim _{k \rightarrow \infty} T\left(t_{k}\right) x$ e então $\mathcal{L}(y)=$ $\lim _{k \rightarrow \infty} \mathcal{L}\left(T\left(t_{k}\right) x\right)=\ell_{+}(x)$. Assim, $\omega(x) \subset \mathcal{Z}$ e $\mathcal{Z}$ atrai $x$. Como $\mathcal{Z}$ é fechado, $\widehat{\mathcal{M}} \subset \mathcal{Z}$. Mas da definição de $\mathcal{Z}$ é claro que $\mathcal{Z} \subset \widehat{\mathcal{M}}$. Portanto $\mathcal{Z}=\widehat{\mathcal{M}}$. Se $\mathcal{Z}$ é um conjunto limitado, então o semigrupo $T(t)$ é pontualmente dissipativo e, se $T(t)$ é limitado, existe um atrator global $\mathcal{M}$, de acordo com o Teorema 2.2. Para cada $x \in \mathcal{M}$, podemos tomar uma órbita completa $\gamma(x)=\{x(t), t \in \mathbb{R}, x(0)=x\} \subset \mathcal{M}$ e definir o conjunto $\alpha$-limite:

$$
\alpha(\gamma(x)):=\bigcap_{\tau \geq 0} \overline{\gamma_{\tau}^{-}(x)}, \text { onde } \gamma_{\tau}^{-}(x):=\{x(t), t \leq \tau\}
$$

Este conjunto $\alpha$-limite é não vazio, invariante e $x(t) \rightarrow \alpha(\gamma(x))$ quando $t \rightarrow-\infty$. Além disso $\alpha(\gamma(x)) \subset \mathcal{Z}$ desde que

$$
\lim _{t \rightarrow-\infty} \mathcal{L}(x(t))=\text { constante }=\left.\mathcal{L}\right|_{\alpha(\gamma(x))}
$$

Agora, se $X$ é conexo e $\mathcal{Z}$ não, então $\mathcal{M}$, que é conexo, contém não somente pontos de $\mathcal{Z}$, mas órbitas completas conectando pontos de $\mathcal{Z}$.

Definição 2.10 Dizemos que um semigrupo $T(t)$ pertence à classe $\mathcal{A} \mathcal{K}$ se para todo conjunto limitado $B \subset X$ tal que $\gamma^{+}(B)$ é limitado, cada sequência da forma

$$
\left\{T\left(t_{k}\right) x_{k}\right\}_{k=1}^{\infty},
$$

onde $x_{k} \in B$ e $t_{k} \rightarrow+\infty$ é pré-compacta.

No que segue estaremos supondo que $T(t)$ é um semigrupo contínuo de classe $\mathcal{A} \mathcal{K}$.

Proposição 5 Para todo conjunto compacto $K$ e $t \in \mathbb{R}^{+}$o conjunto $\gamma_{[0, t]}^{+}(K)$ é pré-compacto.

Prova: $[0, t] \times K$ é compacto, $(t, x) \rightarrow T(t) x$ é contínua. Portanto $\gamma_{[0, t]}^{+}(K)$ é imagem contínua de compacto. 
Proposição 6 Se $K$ é compacto e $\gamma^{+}(K)$ é pré-compacto, então $\omega(K)$ é um conjunto invariante compacto não vazio atraindo $K$.

Prova: Seja $K_{1}:=\overline{\gamma^{+}(K)}$.

$$
T(t)\left(K_{1}\right)=T(t)\left(\overline{\gamma^{+}(K)}\right) \subset \overline{T(t)\left(\gamma^{+}\left(K^{\prime}\right)\right)}=\overline{\gamma_{t}^{+}(K)} \subset \overline{\gamma^{+}(K)}=K_{1} .
$$

Então temos um semigrupo $\left\{T(t), t \in \mathbb{R}^{+}, K_{1}\right\}$ de operadores contínuos $T(t)$ em $K_{1}$. Este semigrupo é de classe $\mathcal{K}$ uma vez que $K_{1}$ é compacto. Como $\gamma^{+}(K)$ é limitado, pelo Teorema $2.1, \omega(K)$ é não vazio, compacto, invariante, e atrai $K$.

Proposição 7 Seja $T(t)$ um semigrupo contínuo de classe $\mathcal{A K}$. Suponhamos que $K$ é um conjunto compacto tal que $\gamma^{+}(K)$ é limitado. Então $\gamma^{+}(K)$ é pré-compacto e portanto $\omega(K)$ é um conjunto nâo vazio, compacto e invariante que atrai $K$.

Prova: Seja $\left\{y_{n}\right\}, n=1,2, \ldots$, uma sequência arbitrária de pontos de $\gamma^{+}(K)$, i. e., $y_{n}=T\left(t_{n}\right) x_{n}, \operatorname{com} x_{n} \in K$ e $t_{n} \in \mathbb{R}^{+}$. Se o conjunto $\left\{t_{n}\right\}_{n=1}^{\infty}$ é limitado, então o conjunto $\left\{y_{n}\right\}_{n=1}^{\infty}$ é pré-compacto pela Proposição 5. Se o conjunto $\left\{t_{n}\right\}_{n=1}^{\infty}$ não é limitado, entâo nós podemos escolher uma subsequência $t_{n_{j}} \rightarrow \infty$ e o conjunto $\left\{T\left(t_{n j}\right) x_{n j}\right\}_{j=1}^{\infty}$ é pré-compacto pois o semigrupo $T(t)$ é de classe $\mathcal{A K}$.

Proposição 8 Seja $T(t)$ um semigrupo contínuo de classe $\mathcal{A K}$. Suponhamos que para algum conjunto limitado $B \subset X, \gamma^{+}(B)$ é limitado. Então $\omega(B)$ é não vazio, compacto, invariante e atrai $B$. Além disso, $\omega(B)$ é conexo se $B$ é conexo.

Prova: Se $x \in B, \gamma^{+}(x)$ é limitado, pois $\gamma^{+}(x) \subset \gamma^{+}(B)$. Então $\omega(x) \neq \emptyset$, e portanto $\omega(B) \neq \emptyset$. Desde que

$$
\omega(B)=\bigcap_{t \geq 0} \overline{\gamma_{t}^{+}(B)} \subset \overline{\gamma^{+}(B)}
$$

é claro que $\omega(B)$ é fechado e limitado. Agora, como os operadores $T(t)$ são contínuos, $T(t)(\omega(B)) \subset \omega(B)$, pois se $x \in \omega(B)$, então

$$
x=\lim _{k \rightarrow \infty} T\left(t_{k}\right) x_{k}, \operatorname{com} x_{k} \in B \text { e } t_{k} \rightarrow \infty .
$$

Portanto

$$
T(t) x=\lim _{k \rightarrow \infty} T\left(t+t_{k}\right) x_{k} \in \omega(B) .
$$

Além disso

$$
T\left(t_{k}\right) x_{k}=T(t)\left(T\left(t_{k}-t\right)\right) x_{k} \text { se } t_{k} \geq t
$$

e o conjunto $\left\{T\left(t_{k}-t\right) x_{k}\right\}_{t_{k} \geq t}$ é pré-compacto já que $T(t)$ é de classe $\mathcal{A} \mathcal{K}$. Assim podemos escolher uma subsequência convergente $\left\{T\left(t_{k_{j}}-t\right) x_{k_{j}}\right\}_{j=1}^{\infty}$ e, se $y=$ $\lim _{j \rightarrow \infty} T\left(t_{k_{j}}-t\right) x_{k_{j}}, y \in \omega(B)$ e $T(t) y=\lim _{j \rightarrow \infty} T\left(t_{k_{j}}\right) x_{k_{j}}=x$. Portanto $x \in$ 
$T(t)(\omega(B))$, logo $\omega(B)=T(t)(\omega(B))$, ou seja, $\omega(B)$ é invariante. Disto segue que cada sequência $\left\{x_{k}\right\}_{k=1}^{\infty}$ com $x_{k} \in \omega(B)$ pode ser representada como $\left\{x_{k}=\right.$ $\left.T(k) \tilde{x}_{k}\right\}_{k=1}^{\infty} \operatorname{com} \tilde{x_{k}} \in \omega(B)$. Logo $\left\{x_{k}\right\}$ é pré-compacto e $\omega(B)$ é compacto.

Resta provar que $\omega(B)$ atrai $B$. Suponhamos que isto não ocorra. Então podemos escolher uma sequência $\left\{T\left(t_{k}\right) x_{k}\right\}_{k=1}^{\infty} \operatorname{com} x_{k} \in B$ e $t_{k} \rightarrow+\infty$ tal que

$$
d\left(\left\{T\left(t_{k}\right) x_{k}\right\}_{k=1}^{\infty}, \omega(B)\right) \geq \varepsilon
$$

para algum $\varepsilon>0$. Como $T(t)$ é de classe $\mathcal{A K},\left\{T\left(t_{k}\right) x_{k}\right\}_{k=1}^{\infty}$ é pré-compacto. Desde que todos os pontos limites do conjunto $\left\{T\left(t_{k}\right) x_{k}\right\}_{k=1}^{\infty}$ devem estar em $\omega(B)$, a distância entre $\omega(B)$ e $\left\{T\left(t_{k}\right) x_{k}\right\}_{k=1}^{\infty}$ é zero, o que contradiz (2.4). De forma análoga à demonstração do Teorema 2.1 mostra-se que $B$ conexo implica $\omega(B)$ conexo.

Teorema 2.4 Seja $T(t)$ um semigrupo continuo limitado e pontualmente dissipativo de classe $\mathcal{A K}$. Então existe um atrator global minimal não vazio $\mathcal{M}$. $\mathcal{M}$ é compacto $e$ invariante e se $X$ é conexo $\mathcal{M}$ também é conexo.

Prova: Pela Proposição 3 existe um conjunto limitado $B_{0}$ tal que $\forall K$ compacto, $\exists \varepsilon_{K}>0$ e $\exists t_{K} \geq 0$ satisfazendo

$$
\begin{gathered}
T(t)\left(\mathcal{O}_{\varepsilon_{K}}(K)\right) \subset B_{0} \quad \forall t \geq t_{K} \\
\text { e } T(t)\left(B_{0}\right) \subset B_{0} \quad \forall t \in \mathbb{R}^{+} .
\end{gathered}
$$

Desde que o semigrupo $T(t)$ é limitado, $\omega\left(B_{0}\right)$ é não vazio, compacto, invariante, e atrai $B_{0}$. Provemos que $\omega\left(B_{0}\right)=\mathcal{M}$. Se $B$ é limitado então $B$ é atraído pelo conjunto compacto $K:=\omega(B)$. Então existe $t_{B_{1}} \geq 0$ talque

$$
\begin{gathered}
T(t)(B) \subset \mathcal{O}_{\varepsilon_{K}}(K) \forall t \geq t_{1}(B) \\
\text { e } T\left(t+t_{1}(B)\right) \subset T(t)\left(\mathcal{O}_{\varepsilon_{K}}(K)\right) \subset B_{0} \quad \forall t \geq t_{K} .
\end{gathered}
$$

Sabemos que $B_{0}$ é atraído para $\omega\left(B_{0}\right)$. Então $B$ é atraído para $\omega\left(B_{0}\right)$ também. De forma análoga à demonstração do Teorema 2.1 , mostra-se que $\omega\left(B_{0}\right)$ é o menor fechado que atrai $B_{0}$, e portanto, $\omega\left(B_{0}\right)=\mathcal{M}$. Se $X$ é conexo, podemos escolher um conjunto conexo limitado $B_{1} \supset B_{0}$. Então $\omega\left(B_{1}\right)$ é conexo, e além disso $\omega\left(B_{1}\right)=$ $\omega\left(B_{0}\right)$. De fato, $\omega\left(B_{0}\right) \subset \omega\left(B_{1}\right)$ pois $\omega\left(B_{0}\right)$ é o menor fechado que atrai $B_{0}$. Da mesma forma, $\omega\left(B_{1}\right) \subset \omega\left(B_{0}\right)$ já que $\omega\left(B_{0}\right)$ atrai $B_{1}$.

Teorema 2.5 Se $T(t)$ é um semigrupo contínuo limitado de classe $\mathcal{A K}$ e possui uma função de Liapunov $\mathcal{L}: X \rightarrow \mathbb{R}$, então todas as afirmações do Teorema 2.3 valem.

A prova deste teorema é essencialmente a mesma do Teorema 2.3, uma vez que temos os resultados do Teorema 2.4 . 
Teorema 2.6 Suponhamos que o semigrupo $T(t)$ está definido em um subconjunto $\mathcal{M}$ de um espaço de Banach $X$ com norma \|\|$X$. Suponhamos também que $T(t)$ pode ser decomposto na soma $W_{t}+U_{t}$, onde $\left\{W_{t}, t \in \mathbb{R}^{+}, \mathcal{M}\right\}$ é uma familia de operadores tal que para todo conjunto limitado $B \in \mathcal{M}$

$$
\left\|W_{t}(B)\right\|_{X} \leq m_{1}(t) m_{2}\left(\|B\|_{X}\right)
$$

onde $m_{i}: \mathbb{R}^{+} \rightarrow \mathbb{R}^{+}$é contínua, $i=1,2, m_{1}(t) \rightarrow 0$ quando $t \rightarrow \infty$, e

$$
\|B\|_{X}:=\sup _{x \in B}\|x\|_{X} .
$$

Se ainda $U_{t}$ é tal que $U_{t}(B)$ é pré-compacto para cada conjunto limitado $B \subset \mathcal{M}$, então $T(t)$ pertence à classe $\mathcal{A} \mathcal{K}$.

Prova: Seja $B$ tal que $\gamma^{+}(B)$ é limitado. Mostraremos que cada conjunto

$$
B_{1}:=\left\{T\left(t_{k}\right) x_{k}\right\}_{k=1}^{\infty}, t_{k} \rightarrow \infty \text { e } x_{k} \in B
$$

pode ser coberto por um número finito de bolas de raio $\varepsilon$, qualquer que seja $\varepsilon>0$. Vamos escolher $\ell$ grande o suficiente para que

$$
m_{1}(\ell) \leq \varepsilon\left[2 m_{2}\left(\|B\|_{X}\right)\right]^{-1}
$$

e decompor $B_{1}$ na soma $B_{1}^{\prime} \cup B_{1}^{\prime \prime}$, onde

$$
\begin{gathered}
B_{1}^{\prime}=\left\{T\left(t_{k}\right) x_{k}\right\}_{k=1}^{k_{1}}, \quad t_{k}<\ell \mathrm{e} \\
B_{1}^{\prime \prime}=\left\{T\left(t_{k}\right) x_{k}\right\}_{k=k_{1}+1}^{\infty} \quad t_{k_{1}+1} \geq \ell .
\end{gathered}
$$

$B_{1}^{\prime \prime}$ é um subconjunto do conjunto $T(\ell)\left(\gamma^{+}(B)\right)$ e qualquer elemento de $T(\ell)\left(\gamma^{+}(B)\right)$ tem a forma $W_{\ell}(x)+U_{\ell}(x), x \in \gamma^{+}(B)$. O conjunto $U_{\ell}\left(\gamma^{+}(B)\right)$ pode ser coberto por um número finito de bolas de raio $\varepsilon / 2$ desde que é pré-compacto, e as normas dos elementos de $W_{\ell}\left(\gamma^{+}(B)\right)$ são menores que $\varepsilon / 2$ pela escolha de $\ell$. Portanto $T(\ell)\left(\gamma^{+}(B)\right)$ pode ser coberto por um número finito de bolas de raio $\varepsilon$, e como $B_{1} \subset T(\ell)\left(\gamma^{+}(B)\right)$ a menos de um número finito de pontos, o mesmo acontece com $B_{1}$. 


\section{Capítulo 3}

\section{Atratores Globais}

\subsection{Introdução}

Neste capítulo mostraremos que a equação das ondas com atrito linear, sob certas condições, dá origem a um semigrupo não linear em $H_{0}^{1}(\Omega) \times L^{2}(\Omega)$ que possui um atrator global minimal. As condições são as seguintes: condição de fronteira de Dirichlet homogênea; termo não linear satisfazendo uma condição de dissipação para garantir a existência global de soluções, limitação e ponto-dissipação, e finalmente o termo não linear deve ainda satisfazer uma condição de crescimento para garantir a existência local de soluções e compacidade assintótica.

Este capítulo está organizado da seguinte maneira: na Seção 2 provamos a existência local de soluções, na Seção 3 mostramos que o semigrupo associado à equação das ondas está globalmente definido e é limitado; por fim, na Seção 4, mostramos que o semigrupo é de classe $\mathcal{A} \mathcal{K}$ e concluímos que este possui um atrator global.

\subsection{Existência Local de Soluções}

Seja $f \in \mathbf{C}^{1}(\mathbb{R}, \mathbb{R})$ satisfazendo:

$$
\begin{aligned}
& \lim _{|s| \rightarrow \infty} \frac{\left|f^{\prime}(s)\right|}{|s|^{2}}=0 \\
& \lim _{|s| \rightarrow \infty} \frac{|f(s)|}{|s|^{3}}=0 .
\end{aligned}
$$


Seja $\Omega$ um subconjunto aberto, limitado e com fronteira suave em $\mathbb{R}^{3}$. Defina $f^{e}(u)=f \circ u$, para $u \in H_{0}^{1}(\Omega)$. Dado $\beta>0$, consideremos o seguinte problema:

$$
u_{t t}+\beta u_{t}=\Delta u+f(u) \quad \text { em } \Omega \subset \mathbb{R}^{3}
$$

com condição de fronteira

$$
u(x)=0 \text { se } x \in \partial \Omega
$$

A equação (3.3) é equivalente ao sistema:

$$
\left\{\begin{array}{l}
u_{t}=v \\
v_{t}=-\beta u_{t}+\Delta u+f(u)
\end{array}\right.
$$

e este, por sua vez, escrevemos na seguinte forma abstrata:

$$
w_{t}=\mathcal{C} w+\mathcal{F}(w)
$$

onde $w=\left[\begin{array}{l}u \\ v\end{array}\right] \in H_{0}^{1}(\Omega) \times \mathrm{L}^{2}(\Omega)=X, \mathcal{C}=\left[\begin{array}{cc}0 & I \\ -\mathcal{A} & -\beta\end{array}\right], \mathcal{F}=\left[\begin{array}{l}0 \\ f^{e}\end{array}\right], \mathrm{e}$

$$
\begin{gathered}
\mathcal{A}: H^{2}(\Omega) \cap H_{0}^{1}(\Omega) \rightarrow L^{2}(\Omega) \\
\mathcal{A} \phi=-\triangle \phi, \quad \forall \phi \in \mathbf{D}(\mathcal{A}) .
\end{gathered}
$$

Nesta seção vamos mostrar que para cada dado inicial $\left(u_{0}, v_{0}\right) \in H_{0}^{1}(\Omega) \times L^{2}(\Omega)$ existe uma solução de $(3.4)(u(t), v(t))$ definida em $\mathbb{R}^{+}$e satisfazendo $(u(0), v(0))=$ $\left(u_{0}, v_{0}\right)=x$, e que esta solução é uma função contínua de $(t, x)$.

Definição 3.1 Sejam $X$ e $Y$ espaços de Banach. Dizemos que uma aplicação $f: X \rightarrow Y$ é limitada se a imagem de qualquer subconjunto limitado de $X$ por $f$ é um subconjunto limitado de $Y$. Dizemos que $f$ é compacta se a imagem de um subconjunto limitado de $X$ por $f$ é um subconjunto pré-compacto de $Y$.

Lema 3.1 Seja $f \in \mathbf{C}^{1}(\mathbb{R}, \mathbb{R})$. Se $u \in H_{0}^{1}(\Omega)$ e $f$ satisfaz (3.2), então $f \circ u \in L^{2}(\Omega)$ e a aplicação

$$
\begin{gathered}
f^{e}: H_{0}^{1}(\Omega) \rightarrow L^{2}(\Omega) \\
f^{e}(u)=f \circ u,
\end{gathered}
$$

é limitada. Adicionalmente, se (3.1) está satisfeita, fe é Lipschitz em conjuntos limitados.

Observamos que é suficiente supor que $f$ satisfaça a condição (3.1), uma vez que esta implica (3.2). 
Prova: Seja $M>0$ tal que $|f(u)| \leq|u|^{3}$ se $|u|>M$, e seja $K_{M}$ tal que $|f(u)| \leq K_{M}$ se $|u| \leq M$. Se

$$
A:=\{x \in \Omega:|u(x)| \leq M\},
$$

então

$$
\begin{aligned}
\left(\int_{\Omega}|f \circ u(x)|^{2} d x\right)^{1 / 2} & =\left(\int_{A}|f \circ u(x)|^{2} d x+\int_{\Omega \backslash A}|f \circ u(x)|^{2} d x\right)^{1 / 2} \\
& \leq\left(\int_{A} K_{M^{2}}^{2} d x+\int_{\Omega}|u(x)|^{6} d x\right)^{1 / 2} \\
& =\left(K_{M}^{2} \mu(A)+\|u\|_{L^{6}}^{6}\right)^{1 / 2} \\
& \leq K_{1}^{\prime}+K_{2}\|u\|_{H^{1}}^{3}<\infty
\end{aligned}
$$

onde $\mu$ é a medida de Lebesgue em $\mathbb{R}^{3}$, e $K_{1}$ e $K_{2}$ são constantes dependendo de $\Omega$. Com isso, se $B \subset H_{0}^{1}(\Omega)$ é limitado, e $K_{B}$ é tal que qualquer que seja $u \in B,\|u\|_{H^{1}} \leq K_{B}$, então

$$
\|f \circ u\|_{L^{2}} \leq K_{1}+K_{2}\|u\|_{H^{1}}^{3} \leq K_{1}+K_{2} K_{B}
$$

Portanto $f^{e}$ é limitada, i.e., a imagem de um subconjunto limitado de $H_{0}^{1}(\Omega)$ por $f^{e}$ é um subconjunto limitado de $L^{2}(\Omega)$. Além disso, Se $\widehat{M}$ é tal que $\left|f^{\prime}(u)\right| \leq|u|^{2}$ para $|u|>\widehat{M}$ e $f^{\prime}(u) \leq K_{\widehat{M}}$ se $u \in[-\widehat{M}, \widehat{M}]$, então

$$
\left|f^{\prime}(u)\right| \leq K_{\widehat{M}}+|u|^{2} \leq K\left(1+|u|^{2}\right), \quad \forall u \in \mathbb{R},
$$

onde $K=\max \left\{1, K_{\widehat{M}}\right\}$. Então dados $u, v \in \mathbb{R}$,

$$
\begin{aligned}
|f(u)-f(v)| & =\left|\int_{u}^{v} f^{\prime}(s) d s\right| \\
& \leq K\left|\int_{u}^{v}\left(1+s^{2}\right) d s\right| \\
& =K\left|v\left(1+\frac{v^{2}}{3}\right)-u\left(1+\frac{u^{2}}{3}\right)\right| \\
& =K|h(u, v)||u-v|,
\end{aligned}
$$

onde

$$
h(u, v)=\frac{v\left(1+\frac{v^{2}}{3}\right)-u\left(1+\frac{u^{2}}{3}\right)}{u-v}=-\frac{1}{3}\left(v^{2}+u^{2}+u v+3\right) .
$$

Notemos que $\left\|h(u, v)^{2}\right\|_{L^{3 / 2}}<\infty$. De fato, $\left\|h(u, v)^{2}\right\|_{L^{3 / 2}}^{3 / 2}=\int_{\Omega}\left|\frac{1}{3}\left(v^{2}(x)+u^{2}(x)+u(x) v(x)+3\right)\right|^{3} d x, \mathbf{e}$ 


$$
\begin{aligned}
\left(v^{2}+u^{2}+u v+3\right)^{3} & =v^{6}+u^{6}+6 v^{4} u^{2}+6 v^{2} u^{4} \\
& +3 u v^{5}+3 u^{5} v+7 u^{3} v^{3}+9 v^{4} \\
& +9 u^{4}+18 u v^{3}+18 u^{3} v+27 v^{2} \\
& +27 u^{2}+27 u^{2} v^{2}+27 u v+27
\end{aligned}
$$

Agora, todos os termos acima têm \|\|$_{L^{1}}$ finita pela desigualdade de Hölder e pelo fato de $H_{0}^{1}(\Omega)$ estar continuamente imerso em $L^{p}(\Omega), p \leq 6$. Além disso, cada um destes termos é majorado por uma constante dependendo de $B$ e de $\Omega$, ou seja, existe $C_{B}$ tal que $\left\|h(u, v)^{2}\right\|_{L^{3 / 2}} \leq C_{B}$. Portanto,

$$
\begin{aligned}
\left\|f^{e}(u)-f^{e}(v)\right\|_{L^{2}}^{2} & =\int_{\Omega}|f(u(x))-f(v(x))|^{2} d x \\
& \leq \int_{\Omega}|h(u(x), v(x))|^{2}|u(x)-v(x)|^{2} d x \\
& \leq\left\|h(u, v)^{2}\right\|_{L^{3 / 2}}\left\|(u-v)^{2}\right\|_{L^{3}} \\
& \leq C_{B}\|u-v\|_{L^{3}}^{2} \\
& \leq L_{B}\|u-v\|_{H^{1}}^{2},
\end{aligned}
$$

onde $L_{B}$ é igual à $C_{B}$ vezes a constante de imersão de $H^{1}(\Omega)$ em $L^{3}(\Omega)$. Como B é um subconjunto limitado arbitrário de $H_{0}^{1}(\Omega)$, concluímos que $f^{e}$ é Lipschitz em conjuntos limitados.

Lema 3.2 Seja $X$ um espaço de Hilbert e $\mathcal{A}$ um operador auto-adjunto densamente definido em $X$, sobrejetor, e satisfazendo

$$
\langle\mathcal{A} x, x\rangle \geq \delta\|x\|^{2} \quad \forall x \in X,
$$

para algum $\delta>0$. Se $\mathcal{C}$ é o operador definido por

$$
\mathcal{C}=\left[\begin{array}{cc}
0 & I \\
-\mathcal{A} & -\beta
\end{array}\right]
$$

$\mathbf{D}(\mathcal{C})=\mathbf{D}(\mathcal{A}) \times X^{1 / 2} \subset X^{1 / 2} \times X=Y$, então $\mathcal{C}$ gera um $C_{0}$ semigrupo em $Y$.

Prova: Como $\overline{\mathbf{D}(\mathcal{A})}^{X^{1 / 2}}=\mathbf{D}\left(\mathcal{A}^{1 / 2}\right)=X^{1 / 2}$ e ${\overline{X^{1 / 2}}}^{X}=X$, é claro que $\overline{\mathbf{D}(\mathcal{C})}=Y$.

Pelo teorema de Lumer-Phillips, basta mostrarmos que $\mathcal{C}$ é dissipativo e existe $\lambda_{0}$ tal que $\mathbf{R}\left(\lambda_{0} I+\mathcal{C}\right)=Y$, onde $\mathbf{R}\left(\lambda_{0} I+\mathcal{C}\right)$ denota a imagem de $\left(\lambda_{0} I+\mathcal{C}\right)$. 
Notemos que $\mathcal{A}$ auto-adjunto implica $\mathcal{A}^{1 / 2}$ auto adjunto. De fato, $R(\lambda:-\mathcal{A})=$ $(\lambda I+\mathcal{A})^{-1}$ é trivialmente auto-adjunto se $\lambda \in \rho(-\mathcal{A}) \cap \mathbb{R}$. Agora,

$$
\mathcal{A}^{-1 / 2}=\frac{1}{\pi} \int_{0}^{\infty} t^{-1 / 2}(t I+\mathcal{A})^{-1} d t
$$

Então

$$
\begin{aligned}
\left\langle\mathcal{A}^{-1 / 2} x, y\right\rangle & =\frac{1}{\pi}\left\langle\int_{0}^{\infty} t^{-1 / 2}(t I+\mathcal{A})^{-1} x d t, y\right\rangle \\
& =\frac{1}{\pi} \int_{0}^{\infty} t^{-1 / 2}\left\langle(t I+\mathcal{A})^{-1} x, y\right\rangle d t \\
& =\frac{1}{\pi} \int_{0}^{\infty} t^{-1 / 2}\left\langle x,(t I+\mathcal{A})^{-1} y\right\rangle d t \\
& =\left\langle x, \frac{1}{\pi} \int_{0}^{\infty} t^{-1 / 2}(t I+\mathcal{A})^{-1} y d t\right\rangle \\
& =\left\langle x, \mathcal{A}^{-1 / 2} y\right\rangle .
\end{aligned}
$$

Portanto, $\mathcal{A}^{-1 / 2}$ é auto-adjunto, e consequentemente $\mathcal{A}^{1 / 2}$ é auto-adjunto.

$Y$ é um espaço de Hilbert com o produto interno

$$
\left\langle\left(x_{1}, x_{2}\right),\left(y_{1}, y_{2}\right)\right\rangle=\left\langle x_{1}, y_{1}\right\rangle_{X^{1 / 2}}+\left\langle x_{2}, y_{2}\right\rangle_{L^{2}}, \quad \forall\left(x_{1}, x_{2}\right),\left(y_{1}, y_{2}\right) \in Y=X^{1 / 2} \times X
$$

e se

$$
x, y \in X^{1 / 2},\langle x, y\rangle_{X^{1 / 2}}=\left\langle\mathcal{A}^{1 / 2} x, \mathcal{A}^{1 / 2} y\right\rangle_{L^{2}} .
$$

A norma em $Y$ proveniente do produto interno $\langle$, $\rangle$ é denotada por \|\| . Se $y \in$ $Y,\|y\|=1$, desde que $Y$ é Hilbert e então uniformemente convexo, o conjunto

$$
F(y):=\left\{y^{*} ; y^{*} \in Y^{*} \mathrm{e}\left\langle y, y^{*}\right\rangle=\|y\|^{2}=\left\|y^{*}\right\|^{2}\right\}
$$

é unitário, ou seja,

$$
F(y)=\{\langle., y\rangle\}
$$

Então se $x \in \mathbf{D}(\mathcal{C})=\mathbf{D}(\mathcal{A}) \times X^{1 / 2}, x=\left(x_{1}, x_{2}\right)$,

$$
\begin{aligned}
\langle\mathcal{C} x, x\rangle & =\left\langle x_{1}, x_{2}\right\rangle_{X^{1 / 2}}-\left\langle\mathcal{A} x_{1}, x_{2}\right\rangle_{L^{2}}-\beta\left\langle x_{2}, x_{2}\right\rangle_{L^{2}} \\
& =\left\langle\mathcal{A}^{1 / 2} x_{1}, \mathcal{A}^{1 / 2} x_{2}\right\rangle_{L^{2}}-\left\langle\mathcal{A} x_{1}, x_{2}\right\rangle_{L^{2}}-\beta\left\langle x_{2}, x_{2}\right\rangle_{L^{2}} \\
& =\left\langle\mathcal{A} x_{1}, x_{2}\right\rangle_{L^{2}}-\left\langle\mathcal{A} x_{1}, x_{2}\right\rangle_{L^{2}}-\beta\left\langle x_{2}, x_{2}\right\rangle_{L^{2}} \\
& =-\beta\left\|x_{2}\right\|_{L^{2}}^{2} \leq 0 .
\end{aligned}
$$

Portanto $\mathcal{C}$ é dissipativo. Além disso, seja $\left(y_{1}, y_{2}\right) \in Y=X^{1 / 2} \times X$ e seja $\lambda_{0}$ tal 
que $\lambda=\lambda_{0}^{2}+\lambda_{0} \beta \in \rho(-\mathcal{A})$. Então $(\lambda I+\mathcal{A})^{-1} \in L(X)$. Logo existe $x_{1} \in \mathbf{D}(\mathcal{A})$ tal que

$$
(\lambda I+\mathcal{A}) x_{1}=\left(\lambda_{0}+\beta\right) y_{1}+y_{2} .
$$

Então

$$
\lambda_{0}^{2} x_{1}+\lambda_{0} \beta x_{1}+\mathcal{A} x_{1}-\lambda_{0} y_{1}-\beta y_{1}=y_{2} .
$$

Se $x_{2}=\lambda_{0} x_{1}-y_{1}$,

$$
\left(\lambda_{0} I-\mathcal{C}\right)\left(x_{1}, x_{2}\right)=\left(\lambda_{0} x_{1}-x_{2},\left(\lambda_{0}+\beta\right) x_{2}+\mathcal{A} x_{1}\right)=\left(y_{1}, y_{2}\right) .
$$

Portanto $\left(\lambda_{0} I+\mathcal{C}\right)$ é sobrejetor e o resultado segue.

Com isso, pelo Teorema 1.14 e o Corolário 1.3 temos que o operador

$$
\mathcal{C}=\left[\begin{array}{cc}
0 & I \\
-\mathcal{A} & -\beta
\end{array}\right]
$$

$\mathbf{D}(\mathcal{C})=H^{2} \cap H_{0}^{1} \times H_{0}^{1} \subset H_{0}^{1} \times L^{2}=Y$, onde $\mathcal{A}$ é o operador

$$
\begin{gathered}
\mathcal{A}: H^{2} \cap H_{0}^{1} \rightarrow L^{2} \\
(\mathcal{A} u) x=-(\triangle u) x
\end{gathered}
$$

gera um $C_{0}$ semigrupo em $Y$. Agora, como $f^{e}$ é Lipschitz em conjuntos limitados, é claro que o mesmo ocorre $\operatorname{com} \mathcal{F}=\left[\begin{array}{c}0 \\ f^{e}\end{array}\right]$.

Assim, pelo Teorema 1.5, para cada valor inicial $w_{0}=\left(u_{0}, v_{0}\right) \in H_{0}^{1}(\Omega) \times L^{2}(\Omega)$ o p.v.i.

$$
\left\{\begin{array}{cl}
w_{t} & =\mathcal{C} w+\mathcal{F}(w) \\
w(0) & =w_{0}
\end{array}\right.
$$

tem uma única solução fraca $w(t)$ cujo intervalo maximal $\left[0, t_{\max }\right)$ de definição é finito somente se

$$
\lim _{t \rightarrow t_{\max }}\|w(t)\|=\infty
$$

Se ainda $\left(u_{0}, v_{0}\right) \in \mathbf{D}(\mathcal{C})$, pelo Teorema $1.7 w(t)$ é solução forte deste p.v.i.. Além disso, as soluções dependem continuamente dos dados iniciais.

Suponhamos que

$$
t_{\max }=\infty, \forall\left(u_{0}, v_{0}\right) \in H_{0}^{1}(\Omega) \times L^{2}(\Omega) .
$$

Então a família de operadores $T(t): H_{0}^{1}(\Omega) \times L^{2}(\Omega) \rightarrow H_{0}^{1}(\Omega) \times L^{2}(\Omega), t \in \mathbb{R}^{+}$

$$
T(t)\left[\begin{array}{l}
u_{0} \\
v_{0}
\end{array}\right]=\left[\begin{array}{l}
u(t) \\
v(t)
\end{array}\right]=e^{\mathcal{c} t}\left[\begin{array}{l}
u_{0} \\
v_{0}
\end{array}\right]+\int_{0}^{t} e^{\mathcal{C}(t-s)} \mathcal{F}\left[\begin{array}{l}
u(s) \\
v(s)
\end{array}\right] d s
$$

define semigrupo em $H_{0}^{1}(\Omega) \times L^{2}(\Omega)$. Mostraremos a seguir que existe uma função de Liapunov associada a $T(t)$. 


\subsection{Existência Global e Limitação}

Nesta seção mostramos que (3.4) define um semigrupo limitado de operadores. Isto é obtido utilizando-se uma função de Liapunov apropriada.

Lema 3.3 Seja $\mathcal{L}: H_{0}^{1}(\Omega) \times L^{2}(\Omega) \rightarrow \mathbb{R}$ definida por:

$$
\mathcal{L}(\phi, \psi)=\frac{1}{2} \int_{\Omega}|\nabla \phi(x)|^{2} d x+\frac{1}{2} \int_{\Omega} \psi^{2}(x) d x-\int_{\Omega} F(u(x)) d x
$$

onde

$$
F(u)=\int_{0}^{u} f(s) d s
$$

e $f$ satisfaz (3.2) e a condição de dissipaçâo :

$$
\varlimsup_{|u| \rightarrow \infty} \frac{f(u)}{u} \leq-\delta<0 .
$$

Então $\mathcal{L}$ é uma função de Liapunov para o semigrupo $T(t)$ definido acima.

Prova: Seja $B$ um subconjunto limitado de $H_{0}^{1}(\Omega) \times L^{2}(\Omega)$, e sejam $\left(\phi_{1}, \psi_{1}\right)$, $\left(\phi_{2}, \psi_{2}\right) \in B$. Existem constantes $C_{1}$ e $C_{2}$ satisfazendo:

$$
\left|\mathcal{L}\left(\phi_{1}, \psi_{1}\right)-\mathcal{L}\left(\phi_{2}, \psi_{2}\right)\right| \leq C_{1}\left\|\phi_{1}-\phi_{2}\right\|_{H_{0}^{1}}+C_{2}\left\|\psi_{1}-\psi_{2}\right\|_{L^{2}}
$$

Para mostrarmos isto basta observarmos que se $\phi_{1}$ e $\phi_{2}$ pertencem a um subconjunto $B_{1}$ de $H_{0}^{1}(\Omega), B_{1}$ limitado, então existe uma constante $L_{B_{1}}$ satisfazendo:

$$
\left|\int_{\Omega} F\left(\phi_{1}(x)\right) d x-\int_{\Omega} F\left(\phi_{2}(x)\right) d x\right| \leq L_{B_{1}}\left\|\phi_{1}-\phi_{2}\right\|_{H_{0}^{1}}
$$

Isso realmente ocorre, pois

$$
\begin{aligned}
\left|F\left(\phi_{1}\right)-F\left(\phi_{2}\right)\right| & =\left|\int_{\phi_{1}}^{\phi_{2}} f(s) d s\right| \leq K\left|\int_{\phi_{1}}^{\phi_{2}}\left(|s|^{4}+1\right) d s\right| \\
& \leq K\left|\phi_{2}-\phi_{1}+\frac{\phi_{2}^{5}}{5}-\frac{\phi_{1}^{5}}{5}\right|=K h\left(\phi_{1}, \phi_{2}\right)\left|\phi_{1}-\phi_{2}\right|,
\end{aligned}
$$

onde $h\left(\phi_{1}, \phi_{2}\right)$ é um polinômio de grau 4 em $\phi_{1}, \phi_{2}$.

Com isso,

$$
\begin{aligned}
\left|\int_{\Omega} F\left(\phi_{1}\right)-F\left(\phi_{2}\right)\right| & \leq \int_{\Omega}\left|h\left(\phi_{1}, \phi_{2}\right) \|\right| \phi_{1}-\phi_{2} \mid \\
& \leq\left\|h\left(\phi_{1}, \phi_{2}\right)\right\|_{L^{6 / 5}}\left\|\phi_{1}-\phi_{2}\right\|_{L^{6}} \\
& \leq\left\|h\left(\phi_{1}, \phi_{2}\right)\right\|_{L^{6 / 5}}\left\|\phi_{1}-\phi_{2}\right\|_{H_{0}^{1}}
\end{aligned}
$$


Agora se $\phi_{1}, \phi_{2} \in B_{1} \subset H_{0}^{1}(\Omega), B_{1}$ limitado, então existe uma constante $L_{B_{1}}$ satisfazendo $(3.6)$ pois

$$
\left\|h\left(\phi_{1}, \phi_{2}\right)\right\|_{L^{6 / 5}} \leq \sum_{i=0}^{4} c_{i}\left\|\phi_{1}^{i} \phi_{2}^{4-i}\right\|_{L^{6 / 5}}+C
$$

e cada um dos termos acima tem norma \|\|$_{L^{1}}$ finita e majorada por uma constante dependendo de $B_{1}$ e de $\Omega$, pela desigualdade de Hölder e pelo fato de $H^{1}(\Omega)$ estar continuamente imerso em $L^{p}(\Omega), p \leq 6$.

Logo temos

$$
\begin{aligned}
\left|\mathcal{L}\left(\phi_{1}, \psi_{1}\right)-\mathcal{L}\left(\phi_{2}, \psi_{2}\right)\right| & \leq\left|\frac{1}{2}\left[\left\|\phi_{1}\right\|_{H_{0}^{1}}^{2}-\left\|\phi_{2}\right\|_{H_{0}^{1}}^{2}\right]\right| \\
& +\left|\frac{1}{2}\left[\left\|\psi_{1}\right\|_{H_{0}^{1}}^{2}-\left\|\psi_{2}\right\|_{H_{0}^{1}}^{2}\right]\right| \\
& +\left|\int_{\Omega}\right| F\left(\phi_{1}\right)-F\left(\phi_{2}\right) \| \mid \\
& \leq C_{1}\left\|\phi_{1}-\phi_{2}\right\|_{H_{0}^{1}}+C_{2}\left\|\psi_{1}-\psi_{2}\right\|_{H_{0}^{1}}
\end{aligned}
$$

ou seja, $\mathcal{L}$ é Lipschitz em conjuntos limitados, logo $\mathcal{L}$ é contínua.

$\mathcal{L}$ é diferenciável quase sempre ao longo de soluções .De fato, se $\left(u_{0}, v_{0}\right) \in D(\mathcal{C})$ temos que

$$
\begin{aligned}
& u_{t t}+\beta u_{t}=\Delta u+f(u) \text { quase sempre em }\left(0, t_{\max }\right) \times \Omega \\
& u=0 \text { em } \partial \Omega, \\
& \mathcal{L}\left(u, u_{t}\right)=\frac{1}{2} \int_{\Omega}|\nabla u(x)|^{2} d x+\frac{1}{2} \int_{\Omega} u_{t}^{2}(x) d x-\int_{\Omega} F(u)(x) d x \\
& \text { e então } \\
& =\frac{1}{2}\langle-\Delta u, u\rangle+\frac{1}{2}\left\langle u_{t}, u_{t}\right\rangle-\langle F(u), 1\rangle, \\
& \frac{d}{d t} \mathcal{L}\left(u, u_{t}\right)=\left\langle-\Delta u, u_{t}\right\rangle+\left\langle u_{t t}, u_{t}\right\rangle-\left\langle f(u), u_{t}\right\rangle \\
& =\left\langle-\Delta u+u_{t t}-f(u), u_{t}\right\rangle \\
& =\left\langle-\beta u_{t}, u_{t}\right\rangle=-\beta\left\|u_{t}\right\|^{2} . \\
& \text { Então se }\left(u_{0}, v_{0}\right) \in \mathbf{D}(\mathcal{C})=\left(H^{2}(\Omega) \cap H_{0}^{1}(\Omega)\right) \times H_{0}^{1}(\Omega) \text {, } \\
& \mathcal{L}\left(T\left(t_{1}\right)\left(u_{0}, v_{0}\right)\right) \leq \mathcal{L}\left(T\left(t_{2}\right)\left(u_{0}, v_{0}\right)\right), \quad t_{1} \geq t_{2} .
\end{aligned}
$$


Como $\mathbf{D}(\mathcal{C})$ é denso em $H_{0}^{1}(\Omega) \times L^{2}(\Omega)$ é claro que $\mathcal{L}$ decresce ao longo de cada órbita e portanto $\mathcal{L}$ é uma função de Liapunov para o semigrupo $T(t)$.

\section{Notemos que}

$$
\lim _{|s| \rightarrow \infty} \frac{|F(s)|}{|s|^{4}}=0
$$

De fato dado $\varepsilon>0$, existe uma constante $c_{\varepsilon}$ tal que

$$
\begin{aligned}
\lim _{|s| \rightarrow \infty} \frac{|F(s)|}{|s|^{4}} & \leq \lim _{|s| \rightarrow \infty} \frac{1}{s^{4}} \int_{0}^{s}|f(t)| d t \\
& \leq \lim _{|s| \rightarrow \infty} \frac{1}{s^{4}} \int_{0}^{s}\left(\varepsilon t^{3}+c_{\varepsilon}\right) d t \\
& =\lim _{|s| \rightarrow \infty} \frac{1}{s^{4}}\left(\frac{\varepsilon s^{4}}{4}+c_{\varepsilon} s\right) \\
& =\lim _{|s| \rightarrow \infty} \frac{\varepsilon}{4}+\frac{c_{\varepsilon}}{s^{3}}=\frac{\varepsilon}{4},
\end{aligned}
$$

se $s>0$, e analogamente se $s<0$. Portanto, como $\Omega$ é limitado, existe uma constante $c$ satisfazendo:

$$
|F(u)| \leq c\left(|u|^{4}+1\right) \text { e } \int_{\Omega}|F(u)| \leq c \int_{\Omega}|u|^{4}+c|\Omega|<\infty
$$

onde $|\Omega|$ denota o volume de $\Omega$. Então

$$
\left|\int_{\Omega} F(\phi)(x) d x\right| \leq c\|\phi\|_{L^{4}}^{4}+c|\Omega| \leq \tilde{c}\|\phi\|_{H_{0}^{1}}^{4}+c|\Omega| .
$$

Logo

$$
\begin{aligned}
|\mathcal{L}(\phi, \psi)| & \leq \frac{1}{2}\|\phi\|_{H_{0}^{1}}^{2}+\frac{1}{2}\|\psi\|_{L^{2}}^{2}+\left|\int_{\Omega} F(\phi)(x) d x\right| \\
& \leq \frac{1}{2}\|\phi\|_{H_{0}^{1}}^{2}+\frac{1}{2}\|\psi\|_{L^{2}}^{2}+\tilde{c}\left|\phi \|_{H_{0}^{1}}^{4}+c\right| \Omega \mid .
\end{aligned}
$$

Por outro lado,

$$
\varlimsup_{\lim _{|u| \rightarrow \infty}} \frac{f(u)}{u}<-\delta \Rightarrow \varlimsup_{\lim } \frac{f(u)+\delta u}{u} \leq 0 .
$$

Então existe $M_{\varepsilon}=M$ tal que $\frac{f(u)+\delta u}{u} \leq \varepsilon$ se $|u| \geq M$, e

$$
\frac{f(u)+\delta u}{u} \leq \varepsilon \quad \text { e } \quad\left[\frac{f(u)+\delta u}{u}-2 \varepsilon\right] \leq-\varepsilon .
$$


Logo,

$\int_{0}^{u} f(s) d s+\frac{\delta u^{2}}{2}-\varepsilon u^{2}=\int_{0}^{u}\left[\frac{f(s)+\delta s}{s}-2 \varepsilon\right] s d s \leq \int_{0}^{M}\left[\frac{f(s)+\delta s}{s}-2 \varepsilon\right] s d s \leq K_{\varepsilon}$, onde $K_{\varepsilon}$ é uma constante dependendo de $\varepsilon$.

Analogamente, se $u>0, u \leq-M$,

$$
\begin{gathered}
{\left[\frac{f(u)+\delta u}{u}-2 \varepsilon\right] \leq-\varepsilon \quad \mathrm{e}} \\
\int_{0}^{u}\left[\frac{f(s)+\delta s}{s}-2 \varepsilon\right] s d s \leq \int_{0}^{-M}\left[\frac{f(s)+\delta s}{s}-2 \varepsilon\right] s d s \leq \widehat{K}_{\varepsilon} .
\end{gathered}
$$

Se $C_{\varepsilon}=\max \left\{K_{\varepsilon}, \widehat{K_{\varepsilon}}\right\}$ temos

$$
\int_{0}^{u} f(s) d s+\frac{\delta u^{2}}{2}-\varepsilon u^{2} \leq C_{\varepsilon}, \quad \forall u \in \mathbb{R} .
$$

Logo,

$$
F(u) \leq C_{\varepsilon}+\varepsilon u^{2}-\frac{\delta u^{2}}{2} \leq C_{\varepsilon}+\varepsilon u^{2} .
$$

Então

$$
\begin{aligned}
\mathcal{L}(\phi, \psi) & \geq \frac{1}{2}\|\phi\|_{H_{0}^{1}}^{2}+\frac{1}{2}\|\psi\|_{L^{2}}^{2}-\int_{\Omega}|F(\phi)(x)| d x \\
& \geq \frac{1}{2}\|\phi\|_{H_{0}^{1}}^{2}+\frac{1}{2}\|\psi\|_{L^{2}}^{2}-\varepsilon\|\phi\|_{L^{2}}^{2}-C_{\varepsilon}|\Omega| \\
& \geq C_{1}\left(\|\phi\|_{H_{0}^{1}}^{2}+\|\psi\|_{L^{2}}^{2}\right)-C_{2},
\end{aligned}
$$

onde $C_{1}$ e $C_{2}$ dependem de $\varepsilon$ e de $\Omega$. Portanto

$$
C_{1}\|(\phi, \psi)\|-C_{2} \leq \mathcal{L}(\phi, \psi) \leq \frac{1}{2}\|\phi\|_{H_{0}^{1}}^{2}+\frac{1}{2}\|\psi\|_{L^{2}}^{2}+\tilde{c}\|\phi\|_{H_{0}^{1}}^{4}+c|\Omega| .
$$

Desde que $\mathcal{L}$ decresce ao longo de soluções,

$$
\mathcal{L}(T(t)(\phi, \psi)) \leq \mathcal{L}(\phi, \psi), \quad \forall(\phi, \psi) \in H_{0}^{1}(\Omega) \times L^{2}(\Omega) .
$$

Concluímos que $T(t)$ é um semigrupo limitado, pois se $B$ é um subconjunto limitado de $H_{0}^{1}(\Omega) \times L^{2}(\Omega)$, e $K_{B}$ é um limitante superior de $\|\phi\|_{H_{0}^{1}},\|\psi\|_{L^{2}},(\phi, \psi) \in B$, então:

$$
C_{1}\|T(t)(\phi, \psi)\|-C_{2} \leq \mathcal{L}(T(t)(\phi, \psi)) \leq \mathcal{L}(\phi, \psi) \leq C_{B},
$$

onde $C_{B}=\frac{1}{2} K_{B}^{2}+\frac{1}{2} K_{B}^{2}+\tilde{c} K_{B}^{4}+c|\Omega|$.

Com isso, é claro que, para cada $\left(u_{0}, v_{0}\right) \in H_{0}^{1}(\Omega) \times L^{2}(\Omega)$, a solução fraca de

$$
\left\{\begin{array}{cl}
w_{t} & =\mathcal{C} w+\mathcal{F}(w) \\
w(0) & =\left(u_{0}, v_{0}\right)
\end{array}\right.
$$


está globalmente definida, pois como já observamos, o intervalo maximal $\left[0, t_{\max }\right)$ de definição da solução $w(t)$ do p.v.i. acima é finito somente se

$$
\lim _{t \rightarrow t_{\max }}\|w(t)\|=\infty
$$

o que não é possível uma vez que $T(t)$ é limitado. Portanto o semigrupo $T(t)$ está bem definido.

\subsection{Compacidade Assintótica e Existência de Atratores Globais}

Nesta seção mostramos que o semigrupo associado a (3.4) é de classe $\mathcal{A} \mathcal{K}$ o que juntamente com os resultados da Seção 3 implicam a existência de um atrator global para o semigrupo associado a (3.4).

Lema 3.4 Se $f$ satisfaz as condiçôes (3.2) e (3.1) e $f^{e}: H_{0}^{1}(\Omega) \rightarrow L^{2}(\Omega)$ é dada por $f^{e}(u)=f \circ u$, então $f^{e}$ é compacta.

Prova: Para mostrarmos que $f^{e}$ é compacta, notamos que por (3.1), dado $\varepsilon>$ $0 \exists M_{\varepsilon}$ tal que se $|u| \geq M_{\varepsilon}$ então $|f(u)| \leq \varepsilon|u|^{3}$. Então

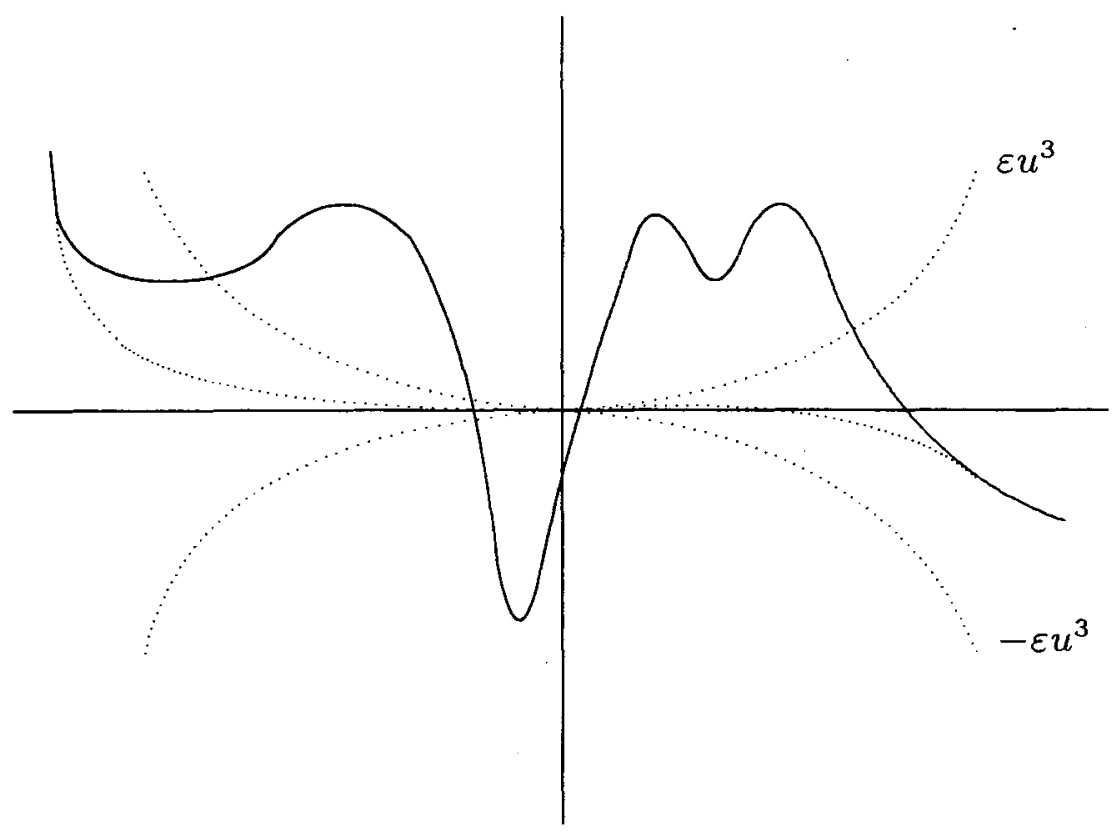




$$
f(u)=\varepsilon g_{\epsilon}(u)+h_{\epsilon}(u),
$$

onde

$$
\left|g_{\varepsilon}(u)\right| \leq|u|^{3},
$$

e $h_{\varepsilon}(u)$ tem suporte compacto. Se $B \subset H_{0}^{1}(\Omega)$ e $v \in f^{e}(B)$, então $v(x)=f^{e}(u)(x)=$ $f \circ u(x), u \in B$ e

$v(x)=f \circ u(x)=\left(\varepsilon g_{\varepsilon}+h_{\varepsilon}\right) \circ u(x)=\varepsilon g_{\varepsilon} \circ u(x)+h_{\varepsilon} \circ u(x)=\varepsilon g_{\varepsilon}^{\varepsilon}(u)(x)+h_{\varepsilon}^{\varepsilon}(u)(x)$.

Logo $f^{e}(B) \subset \varepsilon g_{\varepsilon}^{e}(B)+h_{\varepsilon}^{e}(B)$. Se ainda supomos que as normas dos elementos de $B$ são limitadas por $K_{B}^{\prime}$, então $\forall \varepsilon>0$,

$$
\begin{aligned}
\left\|g_{\varepsilon}^{e}(u)\right\|_{L^{2}}^{2} & =\int_{\Omega}\left|g_{\varepsilon}^{e}(u)(x)\right|^{2} d x=\int_{\Omega}\left|g_{\varepsilon} \circ u(x)\right|^{2} d x \\
& \leq \int_{\Omega}|u(x)|^{6} d x=\|u\|_{L^{6}}^{6} \leq C_{\Omega}\|u\|_{H_{0}^{1}}^{6} \leq C_{\Omega} K_{B}^{6}=C_{B},
\end{aligned}
$$

e $C_{B}$ é uma constante que só depende de $\Omega$ e de $B$. É importante notar que $C_{B}$ não depende de $\varepsilon$. Agora, se $u \in H^{1}(\Omega), h_{\varepsilon}^{\prime}(u) \in L^{\infty}(\Omega)$ pela definição de $h_{\varepsilon}$, e $\nabla u \in L^{2}(\Omega), \log o$

$$
\begin{aligned}
\int_{\Omega}\left|\nabla h_{\varepsilon}^{e}(u)(x)\right|^{2} d x & =\int_{\Omega}\left|h_{\varepsilon}^{\prime}(u)(x) \nabla u(x)\right|^{2} d x \\
& \leq\left\|h_{\varepsilon}^{\prime}(u)\right\|_{L^{\infty}}\|\nabla u\|_{L^{2}},
\end{aligned}
$$

ou seja, $\left\|\nabla h_{\varepsilon}^{e}(u)\right\|_{L^{2}}$ é finita, e ainda mais, a imagem de um conjunto limitado $B \subset H^{1}(\Omega)$ por $h_{\varepsilon}^{e}$ é ainda um subconjunto limitado de $H^{1}(\Omega)$. Como $H^{1}(\Omega)$ está compactamente imerso em $L^{2}(\Omega)$, então $h_{\varepsilon}^{e}(B)$ é pré-compacto em $L^{2}(\Omega)$.

Assim, dado $\delta>0$, seja $\varepsilon$ tal que $\varepsilon . C_{B}=\delta / 3, C_{B}$ como em (3.7). Podemos cobrir $h_{\varepsilon}^{e}(B)$ por um número finito de bolas de raio $\delta / 3$ e centro $x_{i}=h_{\varepsilon}^{e} u_{i}, u_{i} \in B$, $i=1,2, \ldots, r$. Seja $y_{i}=\varepsilon g_{\varepsilon}^{e} u_{i}$ e $w_{i}=x_{i}+y_{i} \in f^{e}(B), i=1,2, \ldots, r$.

$$
\begin{aligned}
\left\|f^{e}(u)-w_{i}\right\|_{L^{2}} & =\left\|\varepsilon g_{\varepsilon}^{e}(u)+h_{\varepsilon}^{e}(u)-x_{i}-y_{i}\right\|_{L^{2}} \\
& \leq\left\|\varepsilon g_{\varepsilon}^{e}(u)\right\|_{L^{2}}+\left\|h_{\varepsilon}^{e}(u)-x_{i}\right\|_{L^{2}}+\left\|y_{i}\right\|_{L^{2}} \\
& \leq \frac{\delta}{3}+\frac{\delta}{3}+\frac{\delta}{3}=\delta,
\end{aligned}
$$

para algum $i, 1 \leq i \leq r$. Portanto, $f^{e}$ é compacta.

Teorema 3.1 O semigrupo $T(t)$ definido em (3.5) é de classe $\mathcal{A} \mathcal{K}$. 
Prova: Seja $U_{t}=e^{\mathcal{c} t}$ e $W_{t}=\int_{0}^{t} e^{\mathcal{C}(t-s)} \mathcal{F}$.

$W_{t}$ é compacta desde que $\mathcal{F}$ é compacta.

Agora, consideremos a equação (3.3) $\operatorname{com} f^{e}=0$,

$$
\phi_{t t}=\Delta \phi-\beta \phi_{t}
$$

que é equivalente à

$$
\left\{\begin{array}{l}
\phi_{t}=\psi \\
\psi_{t}=\Delta \phi-\beta \phi_{t}
\end{array}\right.
$$

e na forma abstrata

$$
w_{t}=\mathcal{C} w
$$

onde $\mathcal{C}=\left(\begin{array}{cc}0 & 1 \\ -\mathcal{A} & -\beta\end{array}\right)$

Pelos Teoremas 1.5 e 1.7 para cada dado inicial $w_{0}=\left(u_{0}, v_{0}\right) \in H_{0}^{1}(\Omega) \times L^{2}(\Omega)$, o p.v.i.

$$
\left\{\begin{array}{cl}
w_{t} & =\mathcal{C} w \quad t \geq 0 \\
w(0) & =\left(u_{0}, v_{0}\right)
\end{array}\right.
$$

tem uma única solução fraca $w(t)$, e se $w_{0} \in \mathbf{D}(\mathcal{C})$, a solução $w(t)$ é forte.

Seja

$$
\mathbf{L}(\phi, \psi)=\frac{1}{2} \int|\nabla \phi|^{2}+\frac{1}{2} \int \psi^{2}+\frac{\delta}{2} \int \phi \psi
$$

Então se $\phi$ satisfaz $\phi_{t t}=\triangle \phi-\beta \phi_{t}$,

$$
\begin{aligned}
\frac{d \mathbf{L}}{d t}\left(\phi, \phi_{t}\right) & =\int_{\Omega} \mathcal{A} \phi \phi_{t}+\frac{\delta}{2} \int_{\Omega}\left(\phi_{t} \phi_{t}+\phi \phi_{t t}\right)+\int_{\Omega} \phi_{t} \phi_{t t} \\
& =-\beta \int_{\Omega} \phi_{t}^{2}+\frac{\delta}{2} \int_{\Omega} \phi_{t}^{2}+\frac{\delta}{2} \int_{\Omega} \phi \phi_{t t} \\
& =\left(-\beta+\frac{\delta}{2}\right) \int_{\Omega} \phi_{t}^{2}+\frac{\delta}{2} \int_{\Omega} \phi\left(-\beta \phi_{t}+\Delta \phi\right) \\
& =\left(-\beta+\frac{\delta}{2}\right) \int_{\Omega} \phi_{t}^{2}-\frac{\delta}{2} \int_{\Omega}|\nabla \phi|^{2}-\frac{\beta \delta}{2} \int_{\Omega} \phi \phi_{t} \\
& =(-2 \beta+\delta) \frac{1}{2} \int_{\Omega} \phi_{t}^{2}-\frac{\delta}{2} \int_{\Omega}|\nabla \phi|^{2}-\frac{\beta \delta}{2} \int_{\Omega} \phi \phi_{t} \\
& \leq-K \mathbf{L}\left(\phi, \phi_{t}\right)
\end{aligned}
$$

onde $K=\min \{(2 \beta-\delta), \delta, \beta\}$. Com isso

$$
\frac{d \mathbf{L}}{d t}\left(\phi, \phi_{t}\right) \leq-K \mathbf{L}\left(\phi, \phi_{t}\right)
$$




$$
\mathbf{L}\left(e^{\mathcal{c} t}\left(u_{0}, v_{0}\right)\right) \leq e^{-K t}
$$

sempre que $\left(u_{0}, v_{0}\right) \in \mathbf{D}(\mathcal{C})$. Segue por densidade que a desigualdade acima também vale em $H_{0}^{1}(\Omega) \times L^{2}(\Omega)$.

Para concluir que o semigrupo gerado por $\mathcal{C}$ decai exponencialmente basta mos trarmos que $\mathbf{L}$ é uma norma equivalente à norma de $H_{0}^{1}(\Omega) \times L^{2}(\Omega)$, o que segue trivialmente da desigualdade de Hölder para $\delta$ suficientemente pequeno.

Para concluir que existe um atrator global para o problema (3.4) falta apenas mostrar que o conjunto dos pontos de equilíbrio de $T(t)$ limitado. De fato, se $\left(u_{0}, v_{0}\right)$ é ponto de equilíbrio de $T(t)$, temos que $v_{0}=0 \mathrm{e}$

$$
\begin{gathered}
-\mathcal{A} u+f^{e}(u)=0 \\
u(\triangle u+f(u))=0 \quad \text { e } \quad \int_{\Omega}|\nabla u|^{2}=\int_{\Omega} f(u) u .
\end{gathered}
$$

Como $\varlimsup_{|u| \rightarrow \infty} \frac{f(u)}{u} \leq-\delta, \frac{f(u)}{u} \leq-\frac{\delta}{2}$, se $|u| \geq M$ para algum $M$. Então

$$
\begin{aligned}
\frac{u^{2} f(u)}{u} & \leq-\frac{\delta}{2}|u|^{2} \quad|u| \geq M \\
\Rightarrow u f(u) & \leq-\frac{\delta}{2}|u|^{2} \quad|u| \geq M \\
\Rightarrow u f(u) & \leq-\frac{\delta}{2}|u|^{2}+\hat{C}_{\delta} \leq \hat{C}_{\delta} \quad \forall u \in \mathbb{R}
\end{aligned}
$$

onde $\widehat{C}_{\delta}$ é tal que $u f(u) \leq \hat{C}_{\delta}$ se $|u| \leq M$. Segue que

$$
\begin{aligned}
\int_{\Omega}|\nabla u|^{2} & =\int_{\Omega} f(u) u \leq \tilde{C}_{\delta}|\Omega|:=C_{\delta}^{2} \quad \mathrm{e} \\
\|u\|_{X^{1 / 2}} & \leq C_{\delta}
\end{aligned}
$$

ou seja, o conjunto dos pontos de equilíbrio é limitado.

Portanto, o problema (3.4) define um semigrupo $T(t)$ contínuo, limitado, de classe $\mathcal{A K}$ que possui uma função de Liapunov $\mathcal{L}$, e além disso, o conjunto $\mathcal{Z}=\{z: T(t) z=z \forall t \geq 0\}$ é limitado. Logo, pelo Teorema 2.5, $T(t)$ possui um atrator global compacto, e todas as afirmações do Teorema 2.3 valem. 


\section{Bibliografia}

[1] J. Arrieta, A. N. Carvalho, J. K. Hale A Damped Hyperbolic Equation whit Critical Exponent, Commun. in Partial Differential Equations, 17(5\& 6), 841-866, [1992].

[2] H. BRÉzIS Análisis Funcional Teoría y Aplicaciones, Alianza Editorial, S. A., Madrid, [1984].

[3] J. K. Hale Asymptotic Behavior of Dissipative Systems, Math. Surveys and Monographs 25, Am. Math. Soc., [1988], Providence, RI.

[4] D. HENRY Evolution Equations in Banach Spaces, Notas não publicadas, IME, USP, [1981], São Paulo, SP.

[5] O. LadYZhenskaya Attractors for Semigroups and Evolution Equations, Cambridge University Press, [1991].

[6] A. Pazy Semigroups of Linear Operators and Aplications to Partial Differential Equations, Applied Mathematical Sciences 44, Springer-Verlag, [1983]. 